\title{
A STABLE PENALTY METHOD FOR THE COMPRESSIBLE NAVIER-STOKES EQUATIONS: III. MULTIDIMENSIONAL DOMAIN DECOMPOSITION SCHEMES*
}

\author{
J. S. HESTHAVEN ${ }^{\dagger}$
}

\begin{abstract}
This paper, concluding the trilogy, develops schemes for the stable solution of wavedominated unsteady problems in general three-dimensional domains. The schemes utilize a spectral approximation in each subdomain and asymptotic stability of the semidiscrete schemes is established. The complex computational domains are constructed by using nonoverlapping quadrilaterals in the two-dimensional case and hexahedrals in the three-dimensional space.

To illustrate the ideas underlying the multidomain method, a stable scheme for the solution of the three-dimensional linear advection-diffusion equation in general curvilinear coordinates is developed. The analysis suggests a novel, yet simple, stable treatment of geometric singularities like edges and vertices. The theoretical results are supported by a two-dimensional implementation of the scheme.

The main part of the paper is devoted to the development of a spectral multidomain scheme for the compressible Navier-Stokes equations on conservation form and a unified approach for dealing with the open boundaries and subdomain boundaries is presented. Well posedness and asymptotic stability of the semidiscrete scheme is established in a general curvilinear volume, with special attention given to a hexahedral domain. The treatment includes a stable procedure for dealing with boundary conditions at a solid wall.

The efficacy of the scheme for the compressible Navier-Stokes equations is illustrated by obtaining solutions to subsonic and supersonic boundary layer flows with various types of boundary conditions. The results are found to agree with the solution of the compressible boundary layer equations.
\end{abstract}

Key words. domain decomposition, stable penalty methods, spectral methods, Navier-Stokes equations

AMS subject classifications. 65M55, 65M70, 65M12, 35Q30

PII. S1064827596299470

1. Introduction. In the first two papers $[1,2]$ of this trilogy, we addressed the problem of open boundary conditions and one-dimensional domain decomposition schemes for the solution of unsteady wave-dominated dissipative problems with the emphasis on asymptotically stable schemes for approximating such problems in fairly simple geometries. In this last paper we relax this restriction and consider multidimensional multidomain schemes for the spectral approximation of wave-dominated problems in general complex geometries.

Although the presentation here is self-contained, the results rely heavily on the previous two papers and in particular on [1] in which much of the theoretical tools are developed. The main purpose of this paper is to devise a multidomain scheme, based on the curvilinear hexahedral as the building block, for the stable solution of the three-dimensional compressible Navier-Stokes equations on conservation form.

Previous work on spectral multidomain methods for the compressible NavierStokes equations is rather sparse. For the spectral multidomain approximation of compressible flows, the emphasis has until recently been on methods for the inviscid Euler equations, where noniterative schemes [3, 4], iterative schemes [5] and spectral

* Received by the editors February 28, 1996; accepted for publication (in revised form) February 21, 1997; published electronically August 5, 1998. This work was supported by NSF grant ASC9504002 and DoE grant DE-FG02-95ER25239.

http://www.siam.org/journals/sisc/20-1/29947.html

${ }^{\dagger}$ Division of Applied Mathematics, Brown University, Box F, Providence, RI 02912 (jansh@ cfm.brown.edu). 
element methods [6] have been proposed. Only recently have several methods for dealing with viscous compressible flows appeared [7, 8, 9, 10]. However, all previous methods for viscous flows are based on a treatment of the inviscid part of the equation, in most cases by applying methods known from the Euler equations, and on a separate treatment of the viscous part of the equation. This second contribution is then applied as a correction to the result obtained from the inviscid patching.

The main difference between previously proposed methods and the one introduced here is that we develop a patching scheme which accounts for the inviscid and viscous part of the equation simultaneously. This approach is made possible by implementing the interface conditions using a penalty term [11, 12], hence allowing for general boundary conditions. In the inviscid limit the proposed algorithm is equivalent to schemes known to perform well when considering the Euler equations. The emphasis is directed toward methods suitable for unsteady flows, and we apply high-order explicit time integration to verify the performance of the scheme.

The remaining part of the paper is organized as follows. In section 2 we introduce several concepts that are crucial to the subsequent analysis. The discussion includes the construction of global maps using transfinite blending functions and the fundamentals of collocation methods using ultraspherical polynomials. Section 3 deals with the multidomain solution of the three-dimensional linear advection-diffusion equation. We propose a semidiscrete scheme for solving this equation and prove that the scheme is asymptotically stable within a curvilinear hexahedral. The theoretical results are supported by numerical studies of the two-dimensional linear advectiondiffusion equation in general geometries. In section 4 we turn the attention toward the development of an asymptotically stable scheme for the compressible Navier-Stokes equations in a general curvilinear hexahedral, which is the fundamental building block of our multidomain scheme. We develop boundary operators ensuring well posedness and prove asymptotic stability of the semidiscrete scheme. Moreover, we also present a stable scheme for imposing boundary conditions at a solid wall. The performance of the scheme is demonstrated by obtaining multidomain solutions to various flat-plate boundary layer flows. Section 5 contains a brief summary and directions for future research.

2. General concepts. The aim of this paper is to devise a general approach for obtaining approximate solutions to wave dominated problems in the general threedimensional domain $\Omega \subset \mathrm{R}^{3}$ enclosed by the boundary $\delta \Omega$. To obtain such solutions we employ polynomial approximations of the unknown functions and their spatial derivatives, with the most natural and computationally efficient extension to several dimensions appearing through the use of tensor products. This procedure, however, requires that the computational domain is diffeomorphic to the unit cube; i.e., a onedomain solution is excluded for general multiply connected domains. To surround this obstruction, we construct $\Omega$ using $K$ nonoverlapping general hexahedrals $\mathrm{D}^{k} \subset \mathrm{R}^{3}$ such that

$$
\Omega=\bigcup_{k=1}^{K} \mathrm{D}^{k}, \delta \Omega=\bigcup_{k=1}^{K} \delta \mathrm{D}_{k k}, \delta \mathrm{D}^{k}=\bigcup_{i=1}^{K} \delta \mathrm{D}_{k i},
$$

where $\delta \mathrm{D}_{k k}=\mathrm{D}^{k} \cap \delta \Omega$ and $\delta \mathrm{D}_{k i}=\mathrm{D}^{k} \cap \mathrm{D}^{i}$ for $i \neq k$. In what remains the emphasis will be on the construction of schemes for solving problems defined in $\mathrm{D}^{k}$. For simplicity, we will refer to any curvilinear hexahedral domain by $\mathrm{D}$ with boundary $\delta \mathrm{D}$, unless clarification is deemed necessary. 
To establish well posedness in D we apply the energy method to prove $L^{2}$-stability. However, to handle general hexahedrals we need to examine a little closer the validity of the integration by parts argument that is so crucial to the energy method. The validity of the multidimensional integration by parts argument hinges on the validity of the divergence theorem. Clearly, if the boundary $\delta \mathrm{D}$ is regular the theorem is valid. However, as established in [13], also in cases where $\delta \mathrm{D}$ is almost smooth may we use integration by parts. Although the outward pointing normal vector $\hat{\boldsymbol{n}}$ remains undefined along the edges and at the vertices of a hexahedral, these geometries have measure zero in $\mathrm{R}^{2}$ and, thus, do not contribute to the boundary term of the partial integration argument establishing that the integration by parts argument remains valid. By domains with almost smooth boundaries we shall understand domains enclosed by boundaries with a finite number of entities having measure zero in $R^{2}$. For a rigorous discussion on the validity of the divergence theorem and an exact definition of almost smooth boundaries, we refer to [13].

2.1. Transfinite blending functions and curvilinear coordinates. To apply the tensor product approximation we require that there exists a diffeomorphism $\Psi: \mathrm{D} \rightarrow \mathrm{I}$, where $\mathrm{I} \subset \mathrm{R}^{3}$ is the unit cube; i.e., $\mathrm{I} \in[-1,1]^{3}$. We will return to the specification of the map $\Psi$ shortly. For convenience, we refer to the coordinates, $\boldsymbol{x} \in \mathrm{D}$, as $(x, y, z)$ and $\left(x_{1}, x_{2}, x_{3}\right)$, interchangeably. Likewise, we introduce the coordinates $\boldsymbol{\xi} \in \mathrm{I}$ named $(\xi, \eta, \zeta)$. The terminology is illustrated in Fig. 1.

For the construction of the map $\Psi: \mathrm{D} \rightarrow \mathrm{I}$, we define $\Xi_{L}=\Xi_{L}^{\xi} \times \Xi_{L}^{\eta} \times \Xi_{L}^{\zeta}$ through the subsets

$$
\Xi_{L}^{\xi}=\left\{\bar{i} \mid \bar{i} \in\left[0, \ldots, L_{\xi}\right]\right\} \quad, \quad \Xi_{L}^{\eta}=\left\{\bar{j} \mid \bar{j} \in\left[0, \ldots, L_{\eta}\right]\right\} \quad, \quad \Xi_{L}^{\zeta}=\left\{\bar{k} \mid \bar{k} \in\left[0, \ldots, L_{\zeta}\right]\right\} .
$$

Associated with these sets are the nodal sets

$$
\Lambda_{L}^{\xi}\left(\Xi_{L}^{\xi}\right)=\left\{\xi_{\bar{i}}\right\}_{\bar{i}=0}^{L_{\xi}}, \Lambda_{L}^{\eta}\left(\Xi_{L}^{\eta}\right)=\left\{\eta_{\bar{j}}\right\}_{\bar{j}=0}^{L_{\eta}}, \Lambda_{L}^{\zeta}\left(\Xi_{L}^{\zeta}\right)=\left\{\zeta_{\bar{k}}\right\}_{\bar{k}=0}^{L_{\zeta}},
$$

with the global nodal set $\Lambda_{L}\left(\Xi_{L}\right)=\Lambda_{L}=\Lambda_{L}^{\xi} \times \Lambda_{L}^{\eta} \times \Lambda_{L}^{\zeta}$. The nodal sets are assumed to be ordered such that $\xi_{0}=\eta_{0}=\zeta_{0}=-1$ and $\xi_{L_{\xi}}=\eta_{L_{\eta}}=\zeta_{L_{\zeta}}=1$. Likewise, for the construction of the polynomial approximation inside the domain I, we introduce the global set $\Xi_{N}=\Xi_{N}^{\xi} \times \Xi_{N}^{\eta} \times \Xi_{N}^{\zeta}$, where

$$
\Xi_{N}^{\xi}=\left\{i \mid i \in\left[0, \ldots, N_{\xi}\right]\right\}, \quad \Xi_{N}^{\eta}=\left\{j \mid j \in\left[0, \ldots, N_{\eta}\right]\right\}, \quad \Xi_{N}^{\zeta}=\left\{k \mid k \in\left[0, \ldots, N_{\zeta}\right]\right\},
$$

with the corresponding nodal sets

$$
\Lambda_{N}^{\xi}\left(\Xi_{N}^{\xi}\right)=\left\{\xi_{i}\right\}_{i=0}^{N_{\xi}}, \Lambda_{N}^{\eta}\left(\Xi_{N}^{\eta}\right)=\left\{\eta_{j}\right\}_{j=0}^{N_{\eta}}, \Lambda_{N}^{\zeta}\left(\Xi_{N}^{\zeta}\right)=\left\{\zeta_{k}\right\}_{k=0}^{N_{\zeta}},
$$

and the global nodal set inside the unit cube $\Lambda_{N}\left(\Xi_{N}\right)=\Lambda_{N}=\Lambda_{N}^{\xi} \times \Lambda_{N}^{\eta} \times \Lambda_{N}^{\zeta}$. The nodes in $\Lambda_{N}$ are assumed ordered as in $\Lambda_{L}$.

For simplicity, we shall use the notation $\Xi_{N}^{\xi \eta}=\Xi_{N}^{\xi} \times \Xi_{N}^{\eta}$ and likewise for various combinations of the sets. For ease of exposure, let us also introduce the global interior set $\tilde{\Xi}_{N}=\tilde{\Xi}_{N}^{\xi} \times \tilde{\Xi}_{N}^{\eta} \times \tilde{\Xi}_{N}^{\zeta} \subset \Xi_{N}$, where

$$
\tilde{\Xi}_{N}^{\xi}=\Xi_{N}^{\xi} \backslash\left\{0, N_{\xi}\right\}, \quad \tilde{\Xi}_{N}^{\eta}=\Xi_{N}^{\eta} \backslash\left\{0, N_{\eta}\right\}, \quad \tilde{\Xi}_{N}^{\zeta}=\Xi_{N}^{\zeta} \backslash\left\{0, N_{\zeta}\right\},
$$

with the associated interior nodal set $\tilde{\Lambda}_{N}$. The exact specification of the nodal sets $\Lambda_{L}$ and $\Lambda_{N}$ shall be addressed shortly. 


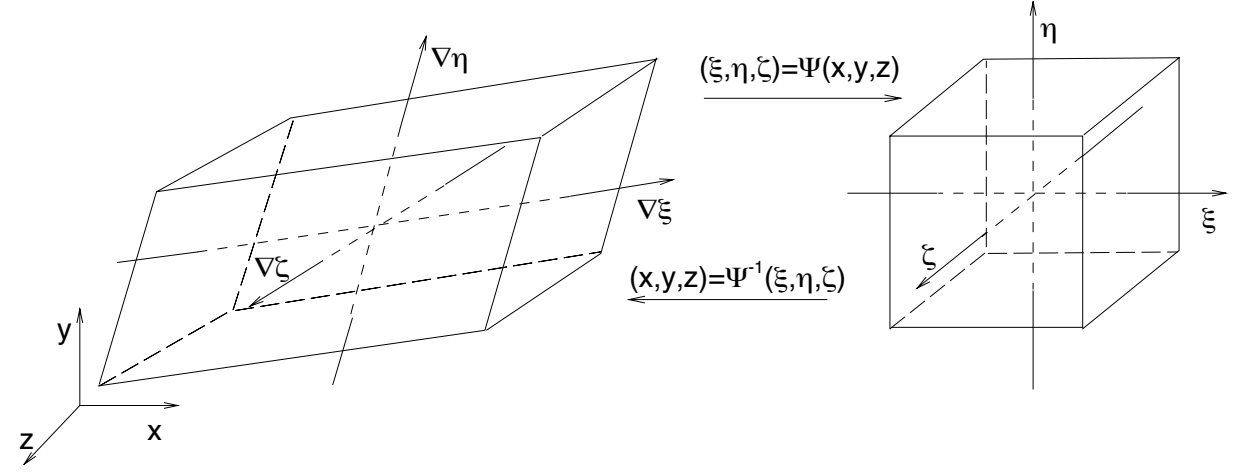

FIG. 1. Three-dimensional mapping between the general hexahedral $\mathrm{D}$ and the unit cube $\mathrm{I}$.

To establish a one-to-one correspondence between the unit cube and the general hexahedral we will construct the global map $\Psi: \mathrm{D} \rightarrow \mathrm{I}$ using transfinite blending functions $[14,15]$. The correspondence between the two domains, expressed through the map $\boldsymbol{x}=\Psi^{-1}(\boldsymbol{\xi})$, is derived from the Boolean sum

(1) $\boldsymbol{x}=\left(P_{\xi} \oplus P_{\eta} \oplus P_{\zeta}\right) \boldsymbol{x}(\boldsymbol{\xi})$

$$
=P_{\xi}(\boldsymbol{x})+P_{\eta}(\boldsymbol{x})+P_{\zeta}(\boldsymbol{x})-P_{\xi} P_{\eta}(\boldsymbol{x})-P_{\xi} P_{\zeta}(\boldsymbol{x})-P_{\eta} P_{\zeta}(\boldsymbol{x})+P_{\xi} P_{\eta} P_{\zeta}(\boldsymbol{x}),
$$

where we have introduced the face projectors

$$
P_{\xi}(\boldsymbol{x})=\sum_{\Xi_{L}^{\xi}} N_{\bar{i}}^{\xi}(\xi) \boldsymbol{x}\left(\xi_{\bar{i}}, \eta, \zeta\right)
$$

and likewise for $P_{\eta}(\boldsymbol{x})$ and $P_{\zeta}(\boldsymbol{x})$. The shape functions $N_{\bar{i}}^{\xi}, N_{\bar{j}}^{\eta}$ and $N_{\bar{k}}^{\zeta}$ are nothing more than the interpolating Lagrange polynomials based on $\Lambda_{L}^{\xi}, \Lambda_{L}^{\eta}$, and $\Lambda_{L}^{\zeta}$, respectively. Using the properties of the projectors, the edge projectors become

$$
P_{\xi} P_{\eta}(\boldsymbol{x})=\sum_{\Xi_{L}^{\xi \eta}} N_{\bar{i}}^{\xi}(\xi) N_{\bar{j}}^{\eta}(\eta) \boldsymbol{x}\left(\xi_{\bar{i}}, \eta_{\bar{j}}, \zeta\right),
$$

and likewise for $P_{\xi} P_{\zeta}(\boldsymbol{x})$ and $P_{\eta} P_{\zeta}(\boldsymbol{x})$, whereas the vertex projector becomes

$$
P_{\xi} P_{\eta} P_{\zeta}(\boldsymbol{x})=\sum_{\Xi_{L}} N_{\bar{i}}^{\xi}(\xi) N_{\bar{j}}^{\eta}(\eta) N_{\bar{k}}^{\zeta}(\zeta) \boldsymbol{x}\left(\xi_{\bar{i}}, \eta_{\bar{j}}, \zeta_{\bar{k}}\right)
$$

To simplify things further we may also apply the transfinite blending function to construct the face projectors as

$$
\begin{aligned}
\boldsymbol{x}\left(\xi_{\bar{i}}, \eta, \zeta\right) & =\left(P_{\eta} \oplus P_{\zeta}\right)\left(\boldsymbol{x}\left(\xi_{\bar{i}}, \eta, \zeta\right)\right) \\
& =P_{\eta}\left(\boldsymbol{x}\left(\xi_{\bar{i}}, \eta, \zeta\right)\right)+P_{\zeta}\left(\boldsymbol{x}\left(\xi_{\bar{i}}, \eta, \zeta\right)\right)-P_{\eta} P_{\zeta}\left(\boldsymbol{x}\left(\xi_{\bar{i}}, \eta, \zeta\right)\right)
\end{aligned}
$$

and similarly for the remaining faces.

Within this formulation, the construction of the global map $\Psi$ or rather its inverse, becomes feasible. In what remains we assume that the shape functions are linear, 
i.e., $L_{\xi}=L_{\eta}=L_{\zeta}=1$ and $\Lambda_{L}^{\xi}=\Lambda_{L}^{\eta}=\Lambda_{L}^{\zeta}=\{-1,1\}$, as $N_{0}^{\xi}=(1-\xi) / 2$ and $N_{1}^{\xi}=(1+\xi) / 2$ and is in a similar fashion for $N_{\bar{j}}^{\eta}$ and $N_{\bar{k}}^{\zeta}$. Thus to construct the map we need to specify a parametric form for the edges enclosing $\mathrm{D}$, e.g.,

$$
\boldsymbol{x}(\xi,-1,-1)=\sum_{\Xi_{N}^{\xi}} \boldsymbol{x}\left(\xi_{i},-1,-1\right) L_{i}^{\alpha}(\xi),
$$

where $L_{i}^{\alpha}(\xi)$ signifies the Lagrange polynomial based on the nodal set $\Lambda_{N}^{\xi}$ employed in I. For a thorough discussion of the transfinite blending functions and their properties, we refer to $[14,15]$.

Once the global map is established, spatial derivatives in $\mathrm{D}$ of the function $f$ are obtained through a general curvilinear coordinate transformation with the metric of the transformation being derived through the identity

$$
\frac{\partial \boldsymbol{x}}{\partial \boldsymbol{\xi}} \frac{\partial \boldsymbol{\xi}}{\partial \boldsymbol{x}}=\left[\begin{array}{ccc}
x_{\xi} & x_{\eta} & x_{\zeta} \\
y_{\xi} & y_{\eta} & y_{\zeta} \\
z_{\xi} & z_{\eta} & z_{\zeta}
\end{array}\right]\left[\begin{array}{lll}
\xi_{x} & \xi_{y} & \xi_{z} \\
\eta_{x} & \eta_{y} & \eta_{z} \\
\zeta_{x} & \zeta_{y} & \zeta_{z}
\end{array}\right]=\mathcal{I},
$$

where $\mathcal{I}$ is the identity matrix, the first Jacobian is obtained from the map, and (1) and the transformation Jacobian becomes

$$
J=\left|\frac{\partial \boldsymbol{x}}{\partial \boldsymbol{\xi}}\right|=x_{\xi} y_{\eta} z_{\zeta}+x_{\zeta} y_{\xi} z_{\eta}+x_{\eta} y_{\zeta} z_{\xi}-x_{\xi} y_{\zeta} z_{\eta}-x_{\eta} y_{\xi} z_{\zeta}-x_{\zeta} y_{\eta} z_{\xi} .
$$

Finally, we note that the local coordinate system in $\mathrm{D}$ is spanned by $(\nabla \xi, \nabla \eta, \nabla \zeta)$ (see Fig. 1), which, in the special case of an orthogonal curvilinear coordinate system, obeys the relation $\nabla \xi \cdot \nabla \eta=\nabla \xi \cdot \nabla \zeta=\nabla \eta \cdot \nabla \zeta=0$.

2.2. Pseudospectral methods. Once we have mapped D onto I, we may proceed by constructing a multidimensional polynomial approximation to a function, $f[\mathrm{I}]: \mathrm{R}^{3} \rightarrow \mathrm{R}$, using a tensor product formulation as

$$
f(\boldsymbol{\xi}) \simeq \sum_{\Xi_{N}} f_{i j k} L_{i}^{\alpha}(\xi) L_{j}^{\alpha}(\eta) L_{k}^{\alpha}(\zeta)
$$

where $f_{i j k}=\left.f\right|_{i j k}=f\left(\xi_{i}, \eta_{j}, \zeta_{k}\right)$ and we have introduced the interpolating Lagrange polynomials $L_{i}^{\alpha}(\xi), L_{j}^{\alpha}(\eta)$, and $L_{k}^{\alpha}(\zeta)$ based on the nodal sets $\Lambda_{N}^{\xi}, \Lambda_{N}^{\eta}$, and $\Lambda_{N}^{\zeta}$, respectively, which are taken to be the Gauss-Lobatto quadrature points; e.g., $\Lambda_{N}^{\xi}$ consists of the roots of the polynomial $\left(1-\xi^{2}\right)\left(P_{N_{\xi}}^{\alpha}\right)^{\prime}(\xi)$, where $P_{N_{\xi}}^{\alpha}$ represents the ultraspherical polynomial of order $N_{\xi}$, and likewise for $\Lambda_{N}^{\eta}$ and $\Lambda_{N}^{\zeta}$.

Associated with the Gauss-Lobatto quadrature points is a Gauss quadrature formula

$$
\sum_{\Xi_{N}} f_{i j k} w_{i}^{\alpha} w_{j}^{\alpha} w_{k}^{\alpha}=\int_{\mathbf{I}} f(\boldsymbol{\xi}) w^{\alpha}(\xi) w^{\alpha}(\eta) w^{\alpha}(\zeta) d \boldsymbol{\xi}
$$

which is exact provided $f(\boldsymbol{\xi})$ is a polynomial of degree less than $2 N_{\xi}$ in $\xi$ and likewise for $\eta$ and $\zeta$. Here, we have the weight functions $w^{\alpha}(\xi), w^{\alpha}(\eta)$, and $w^{\alpha}(\zeta)$ associated with the ultraspherical polynomials and the strictly positive Gauss-Lobatto weights $w_{i}^{\alpha}, w_{j}^{\alpha}$, and $w_{k}^{\alpha}$. The general expressions for the weight functions and the weights are given in [16]. 
To seek approximate solutions to partial differential equations, we need to evaluate spatial derivatives at the nodal set $\Lambda_{N}$. This is accomplished by approximating the continuous operators by differentiation matrices defined at the grid points. The explicit entries of the differentiation matrices can be found in [16]. To compute the multidimensional derivatives one may exploit the tensor-product nature of the approximation, (2), such that derivatives are computed through matrix products.

We shall restrict our attention to two special ultraspherical polynomials: the Legendre polynomials obtained for $\alpha=0$ and the Chebyshev polynomials obtained for $\alpha=-1 / 2$. For further discussions on pseudospectral methods based on Legendre and Chebyshev methods within the present context, we refer to $[1,2]$, and to $[16,17]$ for a more general introduction.

Finally, we introduce some discrete norms that shall be useful in the forthcoming sections. The discrete equivalent of the continuous local Legendre $L^{2}$-norm becomes

$$
\|f\|_{N}^{2}=\sum_{\Xi_{N}}\left(f_{i j k}\right)^{2} J\left(\xi_{i}, \eta_{j}, \zeta_{k}\right) w_{i}^{0} w_{j}^{0} w_{k}^{0},
$$

where $w_{i}^{0}$ represents the Legendre Gauss-Lobatto weights and $J$ is the transformation Jacobian.

To address the accuracy of the schemes we shall use the global discrete weighted Chebyshev $L^{2}$-norm

$$
L_{w}^{2}(f)=\|f\|_{w}=\sum_{K}\left(\frac{\pi^{3}}{N_{\xi} N_{\eta} N_{\zeta}} \sum_{\Xi_{N}} \frac{\left(f_{i j k}\right)^{2} J\left(\xi_{i}, \eta_{j}, \zeta_{k}\right)}{c_{i} c_{j} c_{k}}\right)^{1 / 2},
$$

where $c_{0}=c_{N_{\xi}}=c_{N_{\eta}}=c_{N_{\zeta}}=2$ and $c_{i}=1$ otherwise. This discrete norm is also used for the definition of the global weighted Sobolev norm $H_{w}^{1}$ utilized in the subsequent sections.

3. The advection-diffusion equation. Let us now consider the linear threedimensional advection-diffusion equation

$$
\frac{\partial u}{\partial t}+\nabla \cdot \boldsymbol{F}=\varepsilon \nabla \cdot \boldsymbol{F}_{\varepsilon},
$$

where $u(\boldsymbol{x}, t): \mathbf{D} \times \mathbf{R}_{+} \rightarrow \mathbf{R}, \boldsymbol{F}=[\alpha u, \beta u, \gamma u]^{T}$, and $\boldsymbol{F}_{\varepsilon}=\nabla u$ while $\boldsymbol{V}=(\alpha, \beta, \gamma)$ signifies the constant advective velocity.

Boundary conditions leading to a well-posed problem in a general domain are given in the following lemma.

Lemma 3.1. Assume there exists a solution, $u$, to the three-dimensional linear advection-diffusion equation in a domain $\mathrm{D}$ enclosed by an almost smooth boundary $\delta \mathrm{D}$ with the outward pointing normal vector $\boldsymbol{n}$ uniquely defined at all points of $\delta \mathrm{D}$ with the exception of a finite number of entities having measure zero in $\mathrm{R}^{2}$.

If boundary conditions are given such that

$$
\oint_{\delta \mathrm{D}}\left[-\frac{1}{2} u \boldsymbol{F}+\varepsilon u \boldsymbol{F}_{\varepsilon}\right] \cdot \hat{\boldsymbol{n}} d s \leq 0
$$

the problem is well posed and the solution is bounded as

$$
\frac{1}{2} \frac{d}{d t}\|u\|^{2} \leq-\varepsilon \int_{\mathrm{D}}(\nabla u)^{2} d \boldsymbol{x} .
$$


Proof. Construct the energy integral and apply integration by parts as

$$
\begin{aligned}
\frac{1}{2} \frac{d}{d t}\|u\|^{2} & =-\int_{\mathrm{D}} u \nabla \cdot \boldsymbol{F} d \boldsymbol{x}+\varepsilon \int_{\mathrm{D}} u \nabla \cdot \boldsymbol{F}_{\varepsilon} d \boldsymbol{x} \\
& =\oint_{\delta \mathrm{D}}\left[-\frac{1}{2} u \boldsymbol{F}+\varepsilon u \boldsymbol{F}_{\varepsilon}\right] \cdot \hat{\boldsymbol{n}} d s-\varepsilon \int_{\mathrm{D}} \boldsymbol{F}_{\varepsilon} \cdot \nabla u d \boldsymbol{x}
\end{aligned}
$$

where $\hat{\boldsymbol{n}}$ represents an outward pointing normal vector.

The global condition for stability is satisfied by requiring

$$
\forall \boldsymbol{x} \in \delta \mathrm{D}:-\frac{1}{2 \boldsymbol{V} \cdot \boldsymbol{n}}\left[\left(|\boldsymbol{V} \cdot \boldsymbol{n}| u-\varepsilon \frac{\boldsymbol{V} \cdot \boldsymbol{n}}{|\boldsymbol{V} \cdot \boldsymbol{n}|} \boldsymbol{F}_{\varepsilon} \cdot \boldsymbol{n}\right)^{2}-\left(\varepsilon \boldsymbol{F}_{\varepsilon} \cdot \boldsymbol{n}\right)^{2}\right] \leq 0
$$

where $\boldsymbol{n}=|\boldsymbol{n}| \hat{\boldsymbol{n}}$ is the outward pointing normal vector associated with every point of the boundary. Hence the maximal dissipative boundary operator is given as

$$
\begin{array}{llrl}
\text { Inflow : } & \boldsymbol{V} \cdot \boldsymbol{n} \leq 0: & |\boldsymbol{V} \cdot \boldsymbol{n}| u+\varepsilon \boldsymbol{F}_{\varepsilon} \cdot \boldsymbol{n}=0, \\
\text { Outflow }: & \boldsymbol{V} \cdot \boldsymbol{n} \geq 0: & \varepsilon \boldsymbol{F}_{\varepsilon} \cdot \boldsymbol{n}=0 .
\end{array}
$$

We note that the boundary operator leads to a well-posed problem even in the singular limit of $\varepsilon \rightarrow 0$.

3.1. The semidiscrete scheme. Our objective is to solve (3) using a multidomain method in which $\Omega$ is constructed using a number of hexahedral subdomains D. The penalty methods introduced in $[1,2]$ straightforwardly generalize to multidimensional scalar problems once the proper boundary operator is derived. For simplicity we shall develop the semidiscrete scheme using a Legendre approximation. However, the results extend, with minor modifications, to Chebyshev methods as we will show later.

We use a Legendre collocation method in I and propose to solve

$$
\frac{\partial u}{\partial t}+\nabla \cdot \boldsymbol{F}=\varepsilon \nabla \cdot \boldsymbol{F}_{\varepsilon}-\tau_{i j k} Q(\boldsymbol{x})\left[\alpha_{\mathbf{n}} u(\boldsymbol{x}, t)+\varepsilon G(\boldsymbol{x}, t)-g(\boldsymbol{x}, t)\right]
$$

in each subdomain with $G(\boldsymbol{x}, t)=\boldsymbol{F}_{\varepsilon}(\boldsymbol{x}, t) \cdot \boldsymbol{n}$, and

$$
Q(\boldsymbol{x})= \begin{cases}1 & \text { if } \boldsymbol{x} \in \delta \mathrm{D} \\ 0 & \text { otherwise }\end{cases}
$$

ensuring that the equation is modified at the boundary only.

To construct the proper boundary operator in (4) we introduce

$$
\forall \boldsymbol{x} \in \delta \mathrm{D}: \quad \alpha_{\mathbf{n}}(\boldsymbol{x})= \begin{cases}|\boldsymbol{V} \cdot \boldsymbol{n}| & \text { if } \boldsymbol{V} \cdot \boldsymbol{n} \leq 0 \\ 0 & \text { if } \boldsymbol{V} \cdot \boldsymbol{n} \geq 0\end{cases}
$$

The boundary conditions, be they actual outer boundary conditions or merely conditions required to pass information between subdomains, are given through $g(\boldsymbol{x}, t)$.

To complete the scheme, we need to specify $\tau_{i j k}$ at all boundary points such that the semidiscrete scheme is asymptotically stable. Note that $\tau_{i j k} \equiv 0$ for $\boldsymbol{x} \in \mathrm{D} \backslash \delta \mathrm{D}$.

Lemma 3.2. Assume there exists a solution $u$ to the linear advection-diffusion equation in a curvilinear hexahedral domain $\mathrm{D}$ enclosed by an almost smooth boundary, 
$\delta \mathrm{D}$. Assume also that there exists an diffeomorphism $\Psi: \mathrm{D} \rightarrow \mathrm{I}$, which maps $\mathrm{D}$ onto the unit cube I.

Let $\tau^{ \pm}$be given as

$$
\tau^{ \pm}=\frac{1}{\kappa \tilde{\omega}}\left(1+\kappa \pm \sqrt{1+\left(2+\frac{\boldsymbol{V} \cdot \boldsymbol{n}}{\alpha_{\mathbf{n}}}\right) \kappa}\right) \quad, \quad \kappa=\frac{\varepsilon|\boldsymbol{n}|^{2}}{2 \tilde{\omega} \alpha_{\mathbf{n}}},
$$

with $\boldsymbol{n}$ and $\tilde{\boldsymbol{\omega}}$ being geometry and resolution dependent parameters. The proper choices of these parameters, depending on whether a face, an edge, or a vertex is considered, are given in Appendix A.

If

$$
\forall \boldsymbol{x} \in \delta \mathrm{D}: \tau^{-} \leq \tau_{i j k} \leq \tau^{+},
$$

the scheme (4) is asymptotically stable and the solution is bounded as

$$
\frac{1}{2} \frac{d}{d t}\|u\|_{N}^{2} \leq-\left.\varepsilon \sum_{\tilde{\Xi}_{N}}(\nabla u)^{2}\right|_{i j k} J \omega_{i}^{0} \omega_{j}^{0} \omega_{k}^{0}
$$

Proof. For simplicity, we present the proof for one face, one edge, and one vertex only as the remaining conditions follow from a similar procedure. It is sufficient to consider homogeneous boundary conditions; i.e., $g(\boldsymbol{x}, t)=0$.

To establish the proof, we multiply with $u J \omega_{i}^{0} \omega_{j}^{0} \omega_{k}^{0}$, sum over $\Xi_{N}$, invoke the quadrature rule, and integrate by parts to obtain the requirement for stability

$$
\begin{aligned}
\frac{1}{2} \frac{d}{d t}\|u\|_{N}^{2}= & \oint_{\delta \mathrm{D}}\left[-\frac{1}{2} u \boldsymbol{F}+\varepsilon u \boldsymbol{F}_{\varepsilon}\right] \cdot \hat{\boldsymbol{n}} d s-\varepsilon \int_{\mathrm{D}} \boldsymbol{F}_{\varepsilon} \cdot \nabla u d \boldsymbol{x} \\
& -\sum_{\Xi_{N}} \tau_{i j k} Q(\boldsymbol{x})\left[\alpha_{\mathbf{n}} u^{2}+\varepsilon u G\right] J \omega_{i}^{0} \omega_{j}^{0} \omega_{k}^{0} \leq 0 .
\end{aligned}
$$

Let us for simplicity name the Legendre weights at the endpoints as $\omega_{\xi}, \omega_{\eta}$, and $\omega_{\zeta}$. These can be found in Appendix A. We shall also need the vectors

$$
\boldsymbol{n}_{\xi}=\xi \nabla \xi, \quad \boldsymbol{n}_{\eta}=\eta \nabla \eta, \boldsymbol{n}_{\zeta}=\zeta \nabla \zeta
$$

We now split the treatment into faces, edges, and vertices of the hexahedral.

Face: $(\xi, \eta, \zeta)=(-1, \cdot, \cdot)$ and $\boldsymbol{n}=\boldsymbol{n}_{\xi}=-\nabla \xi$. Collecting the terms that contribute to stability at the face, excluding all edges and vertices, yields the pointwise condition for stability

$\forall(j, k) \in \tilde{\Xi}_{N}^{\eta \zeta}:-\frac{1}{2}\left(\boldsymbol{V} \cdot \boldsymbol{n}+2 \alpha_{\mathbf{n}} \omega_{\xi} \tau_{0 j k}\right) \tilde{u}^{2}+\varepsilon\left(1-\omega_{\xi} \tau_{0 j k}\right) \tilde{u} \tilde{G}-\left.\varepsilon \omega_{\xi}(\nabla u)^{2}\right|_{0 j k} \leq 0$,

since $J, \omega_{j}^{0}$, and $\omega_{k}^{0}$ all are strictly positive. Here $\tilde{u}=u\left(-1, \eta_{j}, \zeta_{k}\right)$ and $\tilde{G}=$ $G\left(-1, \eta_{j}, \zeta_{k}\right)$. By defining the vector $\boldsymbol{R}=(\tilde{u}, \nabla \tilde{u})^{T}$, the question of stability $\boldsymbol{R}^{T} \mathcal{H} \boldsymbol{R} \leq$ 0 , may be recast into choosing the penalty parameter $\tau_{0 j k}$ such that the symmetric matrix $\mathcal{H}$ is negative semidefinite (see [1] for a more detailed discussion of this procedure). The eigenvalue spectrum $\rho(\mathcal{H})$ is $\rho_{1,2}=-\varepsilon \omega_{\xi}$ and

$$
\rho_{3,4}=\frac{1}{2}\left(-\varphi \pm \sqrt{\varphi^{2}-4 \varepsilon\left(-\varepsilon|\boldsymbol{n}|^{2}\left(1-\omega_{\xi} \tau_{0 j k}\right)^{2}+2 \omega_{\xi}\left(2 \alpha_{\mathbf{n}} \omega_{\xi} \tau_{0 j k}+\boldsymbol{V} \cdot \boldsymbol{n}\right)\right)}\right),
$$


where $\varphi=2 \varepsilon \omega_{\xi}+2 \alpha_{\mathbf{n}} \omega_{\xi} \tau_{0 j k}+\boldsymbol{V} \cdot \boldsymbol{n}$. Provided $\varphi>0$, which is guaranteed for $\tau_{0 j k} \geq 1 /\left(2 \omega_{\xi}\right)$, stability is achieved if

$$
\forall(j, k) \in \tilde{\Xi}_{N}^{\eta \zeta}: \quad \varepsilon|\boldsymbol{n}|^{2}\left(1-\omega_{\xi} \tau_{0 j k}\right)^{2}-2 \omega_{\xi}\left(2 \alpha_{\mathbf{n}} \omega_{\xi} \tau_{0 j k}+\boldsymbol{V} \cdot \boldsymbol{n}\right) \leq 0 .
$$

By defining

$$
\tau^{ \pm}=\frac{1}{\kappa \omega_{\xi}}\left(1+\kappa \pm \sqrt{1+\left(2+\frac{\boldsymbol{V} \cdot \boldsymbol{n}}{\alpha_{\mathbf{n}}}\right) \kappa}\right), \quad \kappa=\frac{\varepsilon|\boldsymbol{n}|^{2}}{2 \omega_{\xi} \alpha_{\mathbf{n}}},
$$

stability is guaranteed provided

$$
\forall(j, k) \in \tilde{\Xi}_{N}^{\eta \zeta}: \tau^{-} \leq \tau_{0 j k} \leq \tau^{+} .
$$

Edge: $(\xi, \eta, \zeta)=(-1,-1, \cdot)$ and $\boldsymbol{n}=\omega_{\eta} \boldsymbol{n}_{\xi}+\omega_{\xi} \boldsymbol{n}_{\eta}$. Collecting the terms that contribute to stability along the edge, excluding the vertices, yields the pointwise constraint

$$
\forall k \in \tilde{\Xi}_{N}^{\zeta}:-\frac{1}{2}\left(\boldsymbol{V} \cdot \boldsymbol{n}+2 \alpha_{\mathbf{n}} \tilde{\omega} \tau_{00 k}\right) \tilde{u}^{2}+\varepsilon\left(1-\tilde{\omega} \tau_{00 k}\right) \tilde{u} \tilde{G}-\left.\varepsilon \tilde{\omega}(\nabla u)^{2}\right|_{00 k} \leq 0
$$

where $\tilde{u}=u\left(-1,-1, \zeta_{k}\right)$ and $\tilde{G}=G\left(-1,-1, \zeta_{k}\right)$. We have also introduced the new variable $\tilde{\omega}=\omega_{\xi} \omega_{\eta}$, which appears as a result of the possibility of anisotropic resolution; i.e., $N_{\xi} \neq N_{\eta}$. Consequently, we recover a constraint similar to that obtained on a face and, thus, an equivalent bound on $\tau_{00 k}$ when exchanging $\omega$ with $\tilde{\omega}$ and using the properly defined outward pointing vector. Stability along the remaining edges can be established in a similar manner with the appropriate choices of $\boldsymbol{n}$ and $\tilde{\boldsymbol{\omega}}$ given in Appendix A.

Vertex: $(\xi, \eta, \zeta)=(-1,-1,-1)$ and $\boldsymbol{n}=\omega_{\eta} \omega_{\zeta} \boldsymbol{n}_{\xi}+\omega_{\xi} \omega_{\zeta} \boldsymbol{n}_{\eta}+\omega_{\xi} \omega_{\eta} \boldsymbol{n}_{\zeta}$. Collecting the terms that contribute to stability at the vertex, we recover the pointwise conditions for stability

$$
-\frac{1}{2}\left(\boldsymbol{V} \cdot \boldsymbol{n}+2 \alpha_{\mathbf{n}} \tilde{\omega} \tau_{000}\right) \tilde{u}^{2}+\varepsilon\left(1-\tilde{\omega} \tau_{000}\right) \tilde{u} \tilde{G}-\left.\varepsilon \tilde{\omega}(\nabla u)^{2}\right|_{000} \leq 0,
$$

where $\tilde{u}=u(-1,-1,-1), \tilde{G}=G(-1,-1,-1)$, and $\tilde{\omega}=\omega_{\xi} \omega_{\eta} \omega_{\zeta}$. We recognize this expression as being similar to that arrived at on faces and along edges, thus resulting in a similar stability condition.

The solution is bounded as

$$
\frac{1}{2} \frac{d}{d t}\|u\|_{N}^{2} \leq-\left.\varepsilon \sum_{\tilde{\Xi}_{N}}(\nabla u)^{2}\right|_{i j k} J \omega_{i}^{0} \omega_{j}^{0} \omega_{k}^{0},
$$

establishing asymptotic stability.

A few comments regarding the proof are required. The proof is strictly valid only in the case where the transformation Jacobian $J$ is constant because the proof relies on the exactness of the quadrature rules, which is lost if $J$ is a polynomial of degree higher than zero. If the transformation Jacobian is a constant, implying that $\mathrm{D}$ is a square box, we have $\nabla \xi \cdot \nabla \eta \equiv 0$. This could have been used in the proof to simplify it considerably. However, we choose to maintain the full expressions as we will present results using general curvilinear domains and shall need the full expressions. 
At present, we are not aware of methods by which we can prove the schemes stable if the transformation Jacobian is not constant.

It should also be emphasized that the particularly simple expressions for stability along the edges and at the vertices are a result of the particular choice of the outward pointing vector $\boldsymbol{n}$ at these points. Stability can be established with any choice of $\boldsymbol{n}$ with the only difference being a slightly more complicated expression for $\tau^{ \pm}$as

$$
\tau^{ \pm}=\frac{1}{\kappa \tilde{\omega}}\left(1+\frac{\boldsymbol{n} \cdot \boldsymbol{n}_{\omega}}{|\boldsymbol{n}|^{2}} \kappa \pm \sqrt{\left(1+\frac{\boldsymbol{n} \cdot \boldsymbol{n}_{\omega}}{|\boldsymbol{n}|^{2}} \kappa\right)^{2}+\frac{\boldsymbol{V} \cdot \boldsymbol{n}}{\alpha_{\mathbf{n}}} \kappa-\kappa^{2}}\right),
$$

where $\boldsymbol{n}_{\omega}$ represents the vector introduced in the proof. This $\tau^{ \pm}$results in stability for an arbitrary choice of $\boldsymbol{n}$ along the edges and at the vertices.

We would like to stress that the scheme remains asymptotically stable even in the limit of vanishing diffusivity. Hence even though the scheme is developed for the advection-diffusion equation it may equally well be used for solving multidimensional hyperbolic problems.

3.1.1. Entr'acte on edges and vertices. Although the scheme described in the previous section was proven stable along the edges and at the vertices, there still remains the problem of identifying, among the multitude of solutions available where several domains meet, the principal inflow/outflow domain that holds the proper solution to be imposed at the neighboring edges/vertices.

We define the principal outflow domain as the upstream domain and name the downstream domain as the principal inflow domain. These two domains determine which information should be transferred to the remaining edges/vertices to construct the boundary conditions.

To determining whether a specific edge/vertex belongs to a principal inflow/outflow domain, we recall the three vectors $\boldsymbol{n}_{\xi}, \boldsymbol{n}_{\eta}$, and $\boldsymbol{n}_{\zeta}$ introduced during the proof of Lemma 3.2 .

An edge, say, $(\xi, \eta, \zeta)=(-1,-1, \cdot)$, is identified as a principal outflow edge if $\boldsymbol{V} \cdot \boldsymbol{n}_{\xi}>0$ and $\boldsymbol{V} \cdot \boldsymbol{n}_{\eta}>0$. Inflow is established by reversing both inequalities. Similarly, we identify the principal outflow vertex for $\boldsymbol{V} \cdot \boldsymbol{n}_{\xi}>0, \boldsymbol{V} \cdot \boldsymbol{n}_{\eta}>0$, and $\boldsymbol{V} \cdot \boldsymbol{n}_{\zeta}>0$, where $\boldsymbol{V}$ is the convective velocity vector. The general conditions for this test are summarized in Appendix A.

This rather simple procedure has several advantages. As illustrated in Fig. 2 for an exploded part of a two-dimensional grid with four vertices coming together, this approach uniquely identifies which domain is the principal inflow domain and which is the principal outflow domain. Contrary to previously proposed schemes (see, e.g., $[3,6])$, this scheme can handle any number of domains coming together, as the principal inflow and outflow domains are identified uniquely through the signs of the scalar products.

Once the principal inflow/outflow domains are identified, this process being local to the domain, the state vector is broadcasted to the other elements, and the boundary conditions are enforced. For the boundary conditions of the viscous part, we use the average of the Cartesian derivatives across the edge/vertex. This naturally introduces a small error. However, since the jump in the derivatives decreases at the same order as the schemes, this procedure does not affect the overall accuracy as we shall see shortly. 


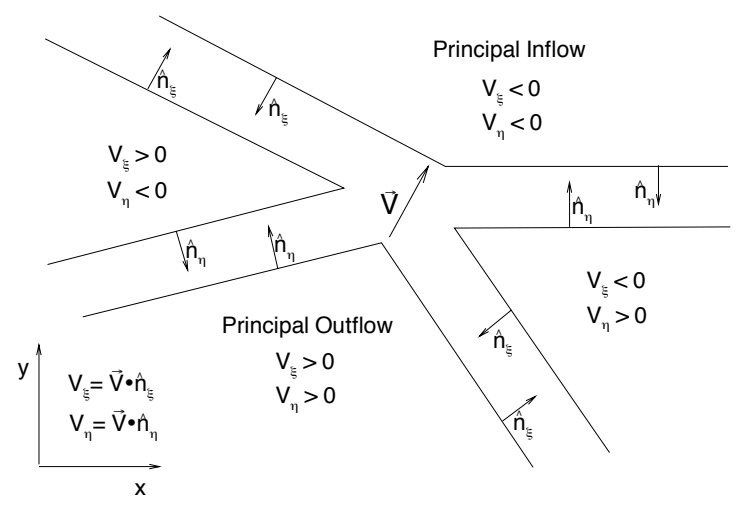

FIG. 2. Illustration of the scheme applied for handling edges/vertices and identifying the principal inflow and outflow domain.

3.2. Examples. We have implemented the proposed scheme (4) for the solution of the two-dimensional linear advection-diffusion equation

$$
\frac{\partial u}{\partial t}+\nabla \cdot \boldsymbol{F}=\varepsilon \nabla \cdot \boldsymbol{F}_{\varepsilon}+f
$$

to study the performance of the scheme. Here $u(x, y, t): \Omega \times \mathrm{R}^{+} \rightarrow \mathrm{R}$ and the force function $f(x, y, t): \Omega \times \mathrm{R}^{+} \rightarrow \mathrm{R}$ is chosen to yield an exact solution as

$$
u_{E}(x, y)=\frac{1}{M} \sum_{i=1}^{M} \exp \left(-\gamma_{i}\left(\left(x-x_{i}\right)^{2}+\left(y-y_{i}\right)^{2}\right)\right)
$$

We have chosen $\Omega=[0,2]^{2}, \boldsymbol{V}=(0.2,0.5)$, and $\varepsilon=0.1$ in all examples. The exact solution (6) is chosen for $M=3$ with $\gamma_{1}=5$ and $\gamma_{2}=\gamma_{3}=10$ and the center of the Gaussians are $\left(x_{1}, y_{1}\right)=(1,0.75),\left(x_{2}, y_{2}\right)=(0.5,1.5)$, and $\left(x_{3}, y_{3}\right)=(1.5,1.5)$. This yields a solution which has advection-dominated regions as well as diffusion-dominated regions within $\Omega$. As initial conditions we used the exact solution perturbed with a two-dimensional cosine. At the open boundary $\delta \Omega$ we enforce the exact solution using the penalty scheme.

We use a collocation method based on Chebyshev polynomials to obtain the approximate solution. Although the scheme (4) was proven stable using Legendre polynomials, stability carries over to the Chebyshev case provided we use the parameters $\omega_{\xi}=N_{\xi}^{-2}$ and $\omega_{\eta}=N_{\eta}^{-2}$. That this choice of $\omega$ leads to a stable approximation was studied extensively in [1] and has recently been proven to be the optimal choice when solving linear hyperbolic systems [18] using Chebyshev methods. Alternatively, the Chebyshev scheme may be proven stable directly by using the technique discussed in [19]. As discussed extensively in [1], the proper choice of the penalty parameter is $\tau_{i j}=\tau_{i j}^{-}$in Lemma 3.2. Moreover, we have reduced $\tau_{i j}$ with a factor of 4 to increase the maximum allowable time-step [1].

Even though we consider a steady-state problem, the scheme was implemented as if the problem is truly unsteady by using a third-order Runge-Kutta method for time integration. In the general three-dimensional case, the time-step is determined 
a)

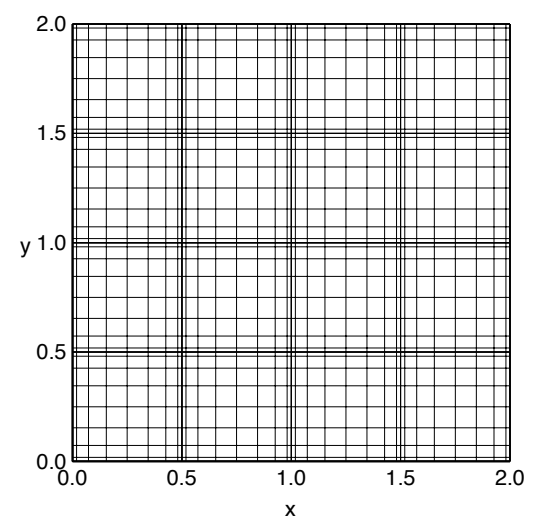

b)

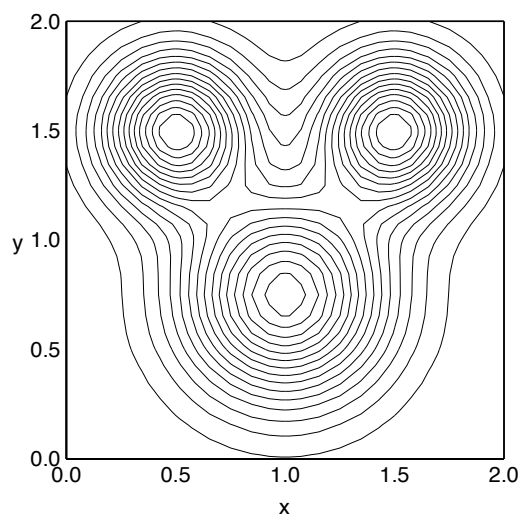

FIG. 3. (a) Grid for $K=16$ and $N_{\xi}=N_{\eta}=8$. (b) Steady-state solution.

by

$$
\Delta t \leq \mathrm{CFL} \times \min _{\mathbf{x} \in \Omega}[|\boldsymbol{\chi} \cdot \boldsymbol{V}|+\varepsilon \boldsymbol{\chi} \cdot \boldsymbol{\chi}]^{-1}
$$

where the local grid distortion vector is given as

$$
\chi=\frac{|\nabla \xi|}{\Delta \xi_{i}}+\frac{|\nabla \eta|}{\Delta \eta_{j}}+\frac{|\nabla \zeta|}{\Delta \zeta_{k}}
$$

with $\Delta \xi_{i}, \Delta \eta_{j}$, and $\Delta \zeta_{k}$ signifying the local grid size. In this particular case, $|\cdot|$ refers to the absolute value of the components; i.e., $|\nabla \xi|=\left(\left|\xi_{x}\right|,\left|\xi_{y}\right|,\left|\xi_{z}\right|\right)$.

We used CFL $=1$ in all test cases, although higher values also lead to stable schemes except for very low resolution. Patching and enforcing of boundary conditions are done at all intermediate time-steps and, following the completion of a time-step, we enforce continuity of the global solution. This is found to stabilize the scheme while maintaining the global spectral accuracy. As a linearization solution at the subdomain boundaries we use the solution obtained at the previous time-step, while the exact solution is used at the open boundaries.

As a first series of tests, we split $\Omega$ into $K$ simple quadrilaterals subdomains D. An example of such a case is shown in Fig. 3, where we illustrate the grid as well as the global solution obtained for $N_{\xi}=N_{\eta}=8$ and $K=16$. As expected from the results obtained in the previous sections, the scheme is stable and we compute the steadystate solution with a global $L^{\infty}$-residual of the order of the machine accuracy. We also note that the solution in Fig. 3(b) is continuous and smooth across the subdomain boundaries.

To assess the global accuracy of the scheme, we vary the number of quadrilateral domains and the order of the polynomial expansion employed in each subdomain. In Fig. 4(a) we plot the global $L^{\infty}$-error as a function of $K$ and $N=N_{\xi}=N_{\eta}$. Indeed, we observe that global spectral convergence is achieved and we observe $p$-convergence (constant $K$ in Fig. 4(a)) as well as $h$-convergence (constant $N$ in Fig. 4(a)).

In [2] we included a brief discussion on the important question of how to minimize the computational work required to ensure a specified global accuracy when using a 
a)

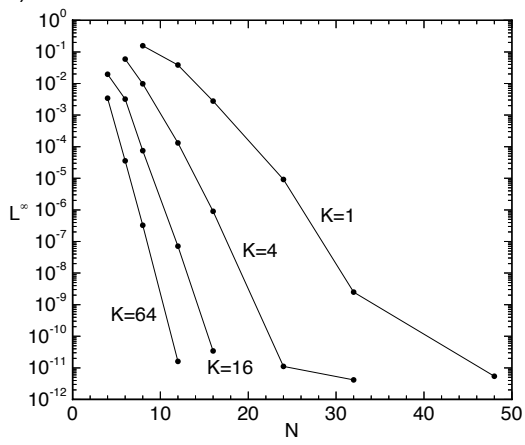

b)

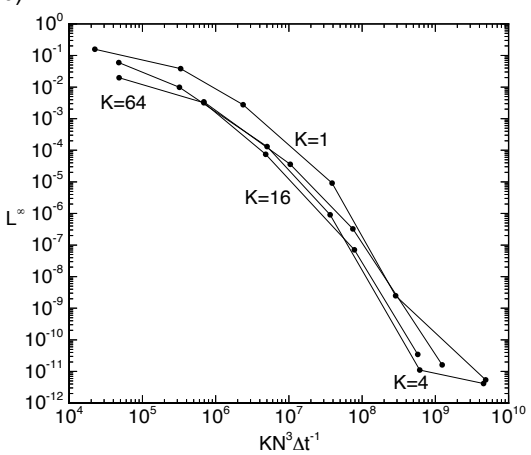

FIG. 4. (a) Global $L^{\infty}$-error for various numbers of subdomains $K$ and the order of the polynomial $N=N_{\xi}=N_{\eta}$ in each subdomain. All subdomains are quadratic as shown in Fig. 3(a). (b) Relation between estimated work to advance one time unit and the corresponding approximation error for different resolution $N=N_{\xi}=N_{\eta}$ and number of subdomains $K$.

multidomain spectral method. However, the analysis was done for the one-dimensional case.

If we restrict the attention to quadrilateral square subdomains with equal order of polynomial expansion $N$ in each direction, we may roughly estimate the work for advancing one time unit as $W=K N^{3} / \Delta t$, where $\Delta t$ is found using (7). In Fig. 4(b) we plot this measure of work against the global $L^{\infty}$-error and observe a behavior similar to that based on the one-dimensional analysis. Indeed, if only low spatial accuracy is required, one should solve the problem by using many subdomains each with a low-order polynomial. On the other hand, if very high spatial accuracy is required, the number of subdomains should be decreased while increasing the order $N$ of the polynomial employed in each subdomain. For the test problem considered here, (6), it appears that $K=16$ represents an optimal number of subdomains over a large range of desired spatial accuracy. These studies indicate that choosing $N \simeq 8-14$ and introducing a sufficient number of subdomains to resolve the problem leads to an acceptable error while minimizing the required computational work.

Although we have no way of proving the scheme stable for subdomains with a nonconstant transformation Jacobian, we apply the scheme for solving the test problem on a distorted grid as shown in Fig. 5(a). The results confirm that the scheme remains stable and maintains global spectral accuracy also on this type of grid. In Fig. 5(b) we compare the global $L^{\infty}$ - and $H_{w}^{1}$-error obtained on the regular grid, Fig. 3(a), with that obtained on the distorted grid. Indeed, we find that spectral accuracy is maintained in $L^{\infty}$ as well as in $H_{w}^{1}$. However, the distortion of the grid leads to a slight decrease of the global accuracy in both norms as compared with the results obtained on the regular grid where the metric is constant. A closer inspection reveals that the loss of accuracy is recovered by increasing the order of the approximating polynomials with one corresponding exactly to the order of the Jacobian, which is a bilinear polynomial for the type of domains considered here. Consequently, the difference in accuracy observed between the two grids is a manifestation of an increased approximation error and not the fact that the patching is performed in the general curvilinear formulation. 
a)

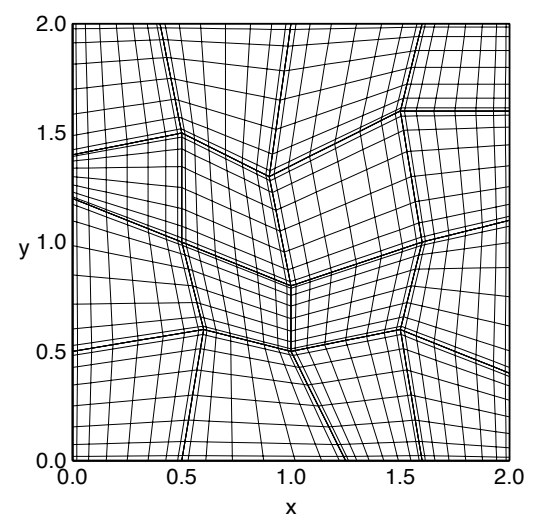

b)

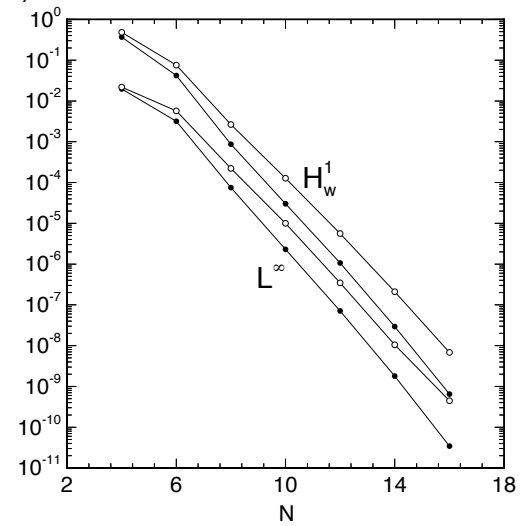

FIG. 5. (a) Distorted grid for $K=16$ and $N=N_{\xi}=N_{\eta}=8$. (b) Comparison between calculated $L^{\infty}$ and $H_{w}^{1}$ as obtained on the grid shown in Fig. 3(a) (solid circles) and the distorted grid shown in (a) (hollow circles).

4. The compressible Navier-Stokes equations. Consider the nondimensional, compressible Navier-Stokes equations on conservation form

$$
\frac{\partial \boldsymbol{q}}{\partial t}+\nabla \cdot \boldsymbol{\Pi}=\frac{1}{\operatorname{Re}_{\mathrm{ref}}} \nabla \cdot \boldsymbol{\Pi}^{\nu} .
$$

The state vector $\boldsymbol{q}=\left(\rho, \rho u_{1}, \rho u_{2}, \rho u_{3}, E\right)^{T}$, the elements of the inviscid flux tensor $\boldsymbol{\Pi}=\left(\boldsymbol{F}_{1}, \boldsymbol{F}_{2}, \boldsymbol{F}_{3}\right)$, and the viscous flux tensor $\boldsymbol{\Pi}^{\nu}=\left(\boldsymbol{F}_{1}^{\nu}, \boldsymbol{F}_{2}^{\nu}, \boldsymbol{F}_{3}^{\nu}\right)$ are given as

$$
\boldsymbol{F}_{i}=\left[\begin{array}{c}
\rho u_{i} \\
\rho u_{1} u_{i}+\delta_{1 i} p \\
\rho u_{2} u_{i}+\delta_{2 i} p \\
\rho u_{3} u_{i}+\delta_{3 i} p \\
(E+p) u_{i}
\end{array}\right], \boldsymbol{F}_{i}^{\nu}=\left[\begin{array}{c}
0 \\
\tau_{x_{1} x_{i}} \\
\tau_{x_{2} x_{i}} \\
\tau_{x_{3} x_{i}} \\
u_{1} \tau_{x_{1} x_{i}}+u_{2} \tau_{x_{2} x_{i}}+u_{3} \tau_{x_{3} x_{i}}+\frac{\gamma k}{\operatorname{Pr}} \frac{\partial T}{\partial x_{i}}
\end{array}\right] .
$$

Here $\delta_{i j}$ is the Kronecker delta function, $\rho$ is the density, $\left(u_{1}, u_{2}, u_{3}\right)=(u, v, w)$ signifies the three Cartesian velocity components, $E$ is the total energy, and $p$ is the pressure. The total energy

$$
E=\rho\left(T+\frac{1}{2}\left(u^{2}+v^{2}+w^{2}\right)\right)
$$

and the pressure $p$ are assumed to be related through the ideal gas law

$$
p=(\gamma-1) \rho T
$$

where $T$ is the temperature field and $\gamma=c_{p} / c_{v}$ is the ratio between the heat capacities at constant pressure $\left(c_{p}\right)$ and volume $\left(c_{v}\right)$, respectively, and is assumed constant.

Considering only Newtonian fluids, the stress tensor elements become

$$
\tau_{x_{i} x_{j}}=\mu\left(\frac{\partial u_{i}}{\partial x_{j}}+\frac{\partial u_{j}}{\partial x_{i}}\right)+\delta_{i j} \lambda \sum_{k=1}^{3} \frac{\partial u_{k}}{\partial x_{k}}
$$


where $\mu$ is the dynamic viscosity, $\lambda$ is the bulk viscosity, and $k$ is the coefficient of thermal conductivity.

The equations are normalized using the reference values $\left(\rho_{\text {ref }}, u_{\text {ref }}, p_{\text {ref }}, T_{\text {ref }}\right)$ as $u_{\text {ref }}=u_{0}, \rho_{\text {ref }}=\rho_{0}, p_{\text {ref }}=\rho_{0} u_{0}^{2}, T_{\text {ref }}=u_{0}^{2} / c_{v}$, and a reference length $L$, where $\left(\rho_{0}, u_{0}\right)$ is a given characteristic state. This yields a Reynolds number as Re $=$ $\rho_{0} u_{0} L / \mu_{0}$ and a Prandtl number as $\operatorname{Pr}=c_{p} \mu_{0} / k_{0}$. Note that the Reynolds number in (9) $\mathrm{Re}_{\mathrm{ref}}$, based on the reference values, in general is different from Re. In what remains we refer to the latter as the Reynolds number. With this normalization we need to specify the Mach number $M$, the Reynolds number Re, the length scale $L$, and a dimensional temperature $T_{0}$.

We consider atmospheric air and take $\gamma=1.4$ and $\operatorname{Pr}=0.72$. To model the temperature dependence of the dynamic viscosity we use Sutherland's viscosity law $[20]$

$$
\frac{\mu(T)}{\mu_{s}}=\left(\frac{T}{T_{s}}\right)^{3 / 2} \frac{T_{s}+S}{T+S}
$$

where $\mu_{s}=1.716 \times 10^{-5} \mathrm{~kg} / \mathrm{m} \mathrm{sec}, T_{s}=273^{\circ} \mathrm{K}$, and $S=110^{\circ} \mathrm{K}$ for atmospheric air. Assuming that the Prandtl number is constant allows for modeling the temperature dependency of the coefficient of thermal conductivity similarly and we adopt Stokes hypothesis (see, e.g., [20]) to obtain $\lambda=-\frac{2}{3} \mu$.

4.1. Well-posed continuous patching conditions. To derive a set of boundary conditions for the constant coefficient compressible Navier-Stokes equations in a general curvilinear volume, we introduce the transformation derivatives

$$
\mathcal{A}_{i}=\frac{\partial \boldsymbol{F}_{i}}{\partial \boldsymbol{q}}, \mathcal{B}_{i j}=\frac{1}{2}\left(\frac{\partial \boldsymbol{F}_{i}^{\nu}}{\partial \boldsymbol{q}_{x_{j}}}+\frac{\partial \boldsymbol{F}_{j}^{\nu}}{\partial \boldsymbol{q}_{x_{i}}}\right), \boldsymbol{q}_{x_{i}}=\frac{\partial \boldsymbol{q}}{\partial x_{i}} .
$$

We shall find it convenient to introduce the operators

$$
\mathcal{A}=\sum_{i=1}^{3} \mathcal{A}_{i} n_{i}, \quad \mathcal{B}_{x}=\sum_{i=1}^{3} \mathcal{B}_{1 i} n_{i}, \quad \mathcal{B}_{y}=\sum_{i=1}^{3} \mathcal{B}_{2 i} n_{i}, \quad \mathcal{B}_{z}=\sum_{i=1}^{3} \mathcal{B}_{3 i} n_{i}
$$

where $\boldsymbol{n}=\left(n_{1}, n_{2}, n_{3}\right)=|\boldsymbol{n}| \hat{\boldsymbol{n}}$ is an outward pointing normal vector of length $|\boldsymbol{n}|$.

Provided the solution $\boldsymbol{q}$ is smooth it is sufficient to consider well posedness and stability for the linearized set of equations as discussed in [21] and applied extensively in $[1,2]$.

A diagonalizing similarity transformation for arbitrary $\boldsymbol{n}$ for the constant coefficient operator $\mathcal{A}\left(\boldsymbol{q}_{0}\right)$ based on primitive variables was derived by Warming, Beam, and Hyett [22] and is given for the conservative formulation in Appendix B. Applying this transformation yields the diagonal matrix $\mathcal{A}^{\mathbf{n}}=\left(\mathcal{S}^{\mathbf{n}}\right)^{-1} \mathcal{A S}^{\mathbf{n}}$ with the diagonal elements

$$
\lambda_{1}^{\mathbf{n}}=\boldsymbol{u}_{0} \cdot \boldsymbol{n}+c_{0}|\boldsymbol{n}|, \quad \lambda_{2}^{\mathbf{n}}=\lambda_{3}^{\mathbf{n}}=\lambda_{4}^{\mathbf{n}}=\boldsymbol{u}_{0} \cdot \boldsymbol{n}, \quad \lambda_{5}^{\mathbf{n}}=\boldsymbol{u}_{0} \cdot \boldsymbol{n}-c_{0}|\boldsymbol{n}|
$$

representing the advective velocities of the characteristic variables $\boldsymbol{R}^{\mathbf{n}}=\left(\mathcal{S}^{\mathbf{n}}\right)^{-1} \boldsymbol{q}$ along $\boldsymbol{n}$. Here $c_{0}=\sqrt{\gamma p_{0} / \rho_{0}}$ represents the sound speed at the linearized state. The explicit form of the characteristic variables $\boldsymbol{R}^{\mathbf{n}}$ is given in Appendix B.

Likewise, we define the transformed viscous matrices

$$
\mathcal{B}_{x}^{\mathbf{n}}=\left(\mathcal{S}^{\mathbf{n}}\right)^{-1} \mathcal{B}_{x} \mathcal{S}^{\mathbf{n}}, \mathcal{B}_{y}^{\mathbf{n}}=\left(\mathcal{S}^{\mathbf{n}}\right)^{-1} \mathcal{B}_{y} \mathcal{S}^{\mathbf{n}}, \mathcal{B}_{z}^{\mathbf{n}}=\left(\mathcal{S}^{\mathbf{n}}\right)^{-1} \mathcal{B}_{z} \mathcal{S}^{\mathbf{n}}
$$


and the viscous correction vector

$$
\boldsymbol{G}^{\mathbf{n}}=\mathcal{B}_{x}^{\mathbf{n}} \frac{\partial \boldsymbol{R}^{\mathbf{n}}}{\partial x}+\mathcal{B}_{y}^{\mathbf{n}} \frac{\partial \boldsymbol{R}^{\mathbf{n}}}{\partial y}+\mathcal{B}_{z}^{\mathbf{n}} \frac{\partial \boldsymbol{R}^{\mathbf{n}}}{\partial z}
$$

with the explicit entries given in Appendix B.

Lemma 4.1. Assume there exists a solution $\boldsymbol{q}$ to the compressible Navier-Stokes equations in a general curvilinear domain D enclosed by an almost smooth boundary $\delta \mathrm{D}$ with the outward pointing normal vector $\boldsymbol{n}$ uniquely defined at all points of $\delta \mathrm{D}$ with the exception of a finite number of entities having measure zero in $\mathrm{R}^{2}$.

Assume also that the fluid properties are constrained as

$$
\mu \geq 0, \quad \lambda \leq 0, \quad \mu+\lambda \geq 0, \frac{\gamma k}{\operatorname{Pr}} \geq 0, \gamma \geq 1 .
$$

Provided the boundary operator is constructed such that

$$
\forall \boldsymbol{x} \in \delta \mathrm{D} \quad \forall i \in[1,5]: \quad R_{i}^{\mathbf{n}}\left[-\frac{1}{2} \lambda_{i}^{\mathbf{n}} R_{i}^{\mathbf{n}}+\frac{1}{\operatorname{Re}_{\mathrm{ref}}} G_{i}^{\mathbf{n}}\right] \leq 0
$$

where $R_{i}^{\mathbf{n}}$ and $G_{i}^{\mathbf{n}}$ represents the components of the vectors $\boldsymbol{R}^{\mathbf{n}}$ and $\boldsymbol{G}^{\mathbf{n}}$, respectively, the constant coefficient problem is well posed and the solution is bounded as

$$
\frac{1}{2} \frac{d}{d t}\left\|\mathcal{Q} \boldsymbol{R}^{\mathbf{i}}\right\|^{2} \leq-\frac{1}{\operatorname{Re}_{\text {ref }}} \int_{\mathrm{D}_{i, j=1}} \sum^{3}\left(\frac{\partial \boldsymbol{R}^{\mathbf{i}}}{\partial x_{i}}\right)^{T} \mathcal{Q}^{T} \mathcal{Q B}_{i j}^{\mathbf{i}} \frac{\partial \boldsymbol{R}^{\mathbf{i}}}{\partial x_{j}} d \boldsymbol{x} \leq 0,
$$

with $\boldsymbol{R}^{\mathbf{i}}=\left(\mathcal{S}^{\mathbf{i}}\right)^{-1} \boldsymbol{q}, \mathcal{B}_{i j}^{\mathbf{i}}=\left(\mathcal{S}^{\mathbf{i}}\right)^{-1} \mathcal{B}_{i j} \mathcal{S}^{\mathbf{i}}$, and $\mathcal{Q}^{T} \mathcal{Q}$ is a positive definite diagonal symmetrizer. Here $\mathbf{i}$ represents the Cartesian unit vector pointing along $x$.

Proof. Consider the constant coefficient matrices, linearized around the uniform state $\boldsymbol{q}_{0}$. In [1] we showed that $\mathcal{A}_{i}$ and $\mathcal{B}_{i j}$ all symmetrize simultaneously using the similarity transform that diagonalizes $\mathcal{A}_{1}$ provided a positive definite diagonal matrix $\mathcal{Q}^{T} \mathcal{Q}=\operatorname{diag}\left(1,2 c_{0}^{2} /\left(\rho_{0}^{2}(\gamma-1)\right), 2,2,1\right)$ is multiplied on from the left.

We use this knowledge and apply the similarity transform $\mathcal{S}^{\mathbf{i}}$ and $\left(\mathcal{S}^{\mathbf{i}}\right)^{-1}$ on the linearized, constant coefficient version of (9), multiply from the left with $\left(\boldsymbol{R}^{\mathbf{i}}\right)^{T} \mathcal{Q}^{T} \mathcal{Q}$, and integrate over $\mathrm{D}$ to obtain the energy integral

$$
\begin{aligned}
\frac{1}{2} \frac{d}{d t}\left\|\mathcal{Q} \boldsymbol{R}^{\mathbf{i}}\right\|^{2}= & -\int_{\mathrm{D}} \sum_{i=1}^{3}\left(\boldsymbol{R}^{\mathbf{i}}\right)^{T} \mathcal{Q}^{T} \mathcal{Q} \mathcal{A}_{i}^{\mathbf{i}} \frac{\partial \boldsymbol{R}^{\mathbf{i}}}{\partial x_{i}} d \boldsymbol{x} \\
& +\frac{1}{\operatorname{Re}_{\text {ref }}} \int_{\mathrm{D}} \sum_{i, j=1}^{3}\left(\boldsymbol{R}^{\mathbf{i}}\right)^{T} \mathcal{Q}^{T} \mathcal{Q} \mathcal{B}_{i j}^{\mathbf{i}} \frac{\partial^{2} \boldsymbol{R}^{\mathbf{i}}}{\partial x_{i} \partial x_{j}} d \boldsymbol{x}
\end{aligned}
$$

where $\mathcal{A}_{i}^{\mathbf{i}}=\left(\mathcal{S}^{\mathbf{i}}\right)^{-1} \mathcal{A}_{i} \mathcal{S}^{\mathbf{i}}$ and $\mathcal{B}_{i j}^{\mathbf{i}}=\left(\mathcal{S}^{\mathbf{i}}\right)^{-1} \mathcal{B}_{i j} \mathcal{S}^{\mathbf{i}}$. The superscript $\mathbf{i}$ indicates that we have applied the similarity transform along the x-axis; i.e., $\mathcal{A}_{1}$ is diagonal. As all the operators by now are symmetric [1], we apply integration by parts to obtain

$$
\begin{aligned}
\frac{1}{2} \frac{d}{d t}\left\|\mathcal{Q} \boldsymbol{R}^{\mathbf{i}}\right\|^{2}= & \oint_{\delta \mathrm{D}}\left(\boldsymbol{R}^{\mathbf{i}}\right)^{T} \mathcal{Q}^{T} \mathcal{Q}\left(\sum_{i=1}^{3}\left[-\frac{1}{2} \mathcal{A}_{i}^{\mathbf{i}} \boldsymbol{R}^{\mathbf{i}}+\frac{1}{\operatorname{Re}_{\mathrm{ref}}} \sum_{j=1}^{3} \mathcal{B}_{i j}^{\mathbf{i}} \frac{\partial \boldsymbol{R}^{\mathbf{i}}}{\partial x_{j}}\right] n_{i}\right) d s \\
& -\frac{1}{\operatorname{Re}_{\text {ref }}} \int_{\mathrm{D}} \sum_{i, j=1}^{3}\left(\frac{\partial \boldsymbol{R}^{\mathbf{i}}}{\partial x_{i}}\right)^{T} \mathcal{Q}^{T} \mathcal{Q} \mathcal{B}_{i j}^{\mathbf{i}} \frac{\partial \boldsymbol{R}^{\mathbf{i}}}{\partial x_{j}} d \boldsymbol{x}
\end{aligned}
$$


If we first consider the remaining volume integral, we established in [1] that it is positive semidefinite provided the properties of the fluid are restricted as

$$
\mu \geq 0, \quad \lambda \leq 0, \quad \mu+\lambda \geq 0, \frac{\gamma k}{\operatorname{Pr}} \geq 0, \gamma \geq 1 .
$$

The boundary integral may be made negative by requiring

$$
\forall \boldsymbol{x} \in \delta \mathrm{D}:\left(\boldsymbol{R}^{\mathbf{i}}\right)^{T} \mathcal{Q}^{T} \mathcal{Q}\left(\sum_{i=1}^{3}\left[-\frac{1}{2} \mathcal{A}_{i}^{\mathbf{i}} \boldsymbol{R}^{\mathbf{i}}+\frac{1}{\operatorname{Re}_{\mathrm{ref}}} \sum_{j=1}^{3} \mathcal{B}_{i j}^{\mathbf{i}} \frac{\partial \boldsymbol{R}^{\mathbf{i}}}{\partial x_{j}}\right] n_{i}\right) \leq 0 .
$$

The problem with this formulation is that $\mathcal{A}_{1}^{\mathrm{i}}$ rather than the appropriate $\mathcal{A}^{\mathbf{n}}$ is diagonal, thus making it impossible to derive the required boundary operator. To obtain the correct formulation, we introduce the operator $\mathcal{H}^{\mathbf{i}, \mathbf{n}}=\left(\mathcal{S}^{\mathbf{i}}\right)^{-1} \mathcal{S}^{\mathbf{n}}$ by which we may recast the condition as

$$
\forall \boldsymbol{x} \in \delta \mathrm{D}: \quad\left(\boldsymbol{R}^{\mathbf{n}}\right)^{T}\left(\mathcal{H}^{\mathbf{i}, \mathbf{n}}\right)^{T} \mathcal{Q}^{T} \mathcal{Q} \mathcal{H}^{\mathbf{i}, \mathbf{n}}\left[-\frac{1}{2} \mathcal{A}^{\mathbf{n}} \boldsymbol{R}^{\mathbf{n}}+\frac{1}{\operatorname{Re}_{\mathrm{ref}}} \boldsymbol{G}^{\mathbf{n}}\right] \leq 0
$$

such that $\mathcal{A}^{\mathbf{n}}$ becomes diagonal. We observe that the operator $\left(\mathcal{H}^{\mathbf{i}, \mathbf{n}}\right)^{T} \mathcal{Q}^{T} \mathcal{Q H}^{\mathbf{i}, \mathbf{n}}$ is symmetric and, using Sylvester's law of inertia for symmetric matrices [23], is positive definite since $\mathcal{Q}^{T} \mathcal{Q}$ is positive definite. Consequently, a pointwise boundary operator leading to a well-posed problem may be constructed componentwise as

$$
\forall \boldsymbol{x} \in \delta \mathrm{D}, \forall i \in[1,5]: \quad R_{i}^{\mathbf{n}}\left[-\frac{1}{2} \lambda_{i}^{\mathbf{n}} R_{i}^{\mathbf{n}}+\frac{1}{\operatorname{Re}_{\mathrm{ref}}} G_{i}^{\mathbf{n}}\right] \leq 0,
$$

where $R_{i}^{\mathbf{n}}$ and $G_{i}^{\mathbf{n}}$ represent the components of the vectors $\boldsymbol{R}^{\mathbf{n}}$ and $\boldsymbol{G}^{\mathbf{n}}$.

To derive a more appropriate form of the boundary operator, we express the condition for stability along $\boldsymbol{n}$ as

$$
-\frac{1}{2}\left[\sum_{i=1}^{5}\left(\lambda_{i}^{\mathbf{n}}\right)^{-1}\left(\left(\left|\lambda_{i}^{\mathbf{n}}\right| R_{i}^{\mathbf{n}}-\varepsilon \frac{\lambda_{i}^{\mathbf{n}}}{\left|\lambda_{i}^{\mathbf{n}}\right|} G_{i}^{\mathbf{n}}\right)^{2}-\left(\varepsilon G_{i}^{\mathbf{n}}\right)^{2}\right)\right] \leq 0,
$$

where $\varepsilon=1 / \operatorname{Re}_{\text {ref }}$. We recall that $\lambda_{i}^{\mathbf{n}}$ represents the characteristic velocity by which the characteristic variable $R_{i}^{\mathbf{n}}$ is advected.

The maximal dissipative boundary conditions are thus given on the form

$$
\mathcal{R}_{ \pm}^{\mathbf{n}} \boldsymbol{R}^{\mathbf{n}}+\frac{1}{\mathrm{Re}_{\mathrm{ref}}} \mathcal{G}_{ \pm} \boldsymbol{G}^{\mathbf{n}}=0
$$

For the inflow case $\boldsymbol{u}_{0} \cdot \boldsymbol{n}<0$, it is constructed by using

$$
\mathcal{R}_{-}^{\mathbf{n}}=\left[\begin{array}{ccccc}
\alpha\left|\lambda_{1}^{\mathbf{n}}\right| & 0 & 0 & 0 & 0 \\
0 & \left|\lambda_{2}^{\mathbf{n}}\right| & 0 & 0 & 0 \\
0 & 0 & \left|\lambda_{3}^{\mathbf{n}}\right| & 0 & 0 \\
0 & 0 & 0 & \left|\lambda_{4}^{\mathbf{n}}\right| & 0 \\
0 & 0 & 0 & 0 & \left|\lambda_{5}^{\mathbf{n}}\right|
\end{array}\right] \quad, \quad \mathcal{G}_{-}=\left[\begin{array}{ccccc}
1 & 0 & 0 & 0 & 0 \\
0 & 1 & 0 & 0 & 0 \\
0 & 0 & 1 & 0 & 0 \\
0 & 0 & 0 & 1 & 0 \\
0 & 0 & 0 & 0 & 1
\end{array}\right],
$$

with $\alpha=0$ for subsonic conditions and $\alpha=1$ for supersonic conditions. Likewise, for the outflow case $\boldsymbol{u}_{0} \cdot \boldsymbol{n}>0$, the boundary operator appears by using

$$
\mathcal{R}_{+}^{\mathbf{n}}=\left[\begin{array}{ccccc}
0 & 0 & 0 & 0 & 0 \\
0 & 0 & 0 & 0 & 0 \\
0 & 0 & 0 & 0 & 0 \\
0 & 0 & 0 & 0 & 0 \\
0 & 0 & 0 & 0 & \beta\left|\lambda_{5}^{\mathbf{n}}\right|
\end{array}\right], \quad \mathcal{G}_{+}=\left[\begin{array}{ccccc}
0 & 0 & 0 & 0 & 0 \\
0 & 1 & 0 & 0 & 0 \\
0 & 0 & 1 & 0 & 0 \\
0 & 0 & 0 & 1 & 0 \\
0 & 0 & 0 & 0 & 1
\end{array}\right],
$$


with $\beta=1$ for subsonic conditions and $\beta=0$ for supersonic outflow conditions.

The singular nature of $\mathcal{G}_{+}$is a consequence of $G_{1}^{\mathbf{n}}=-G_{5}^{\mathbf{n}}$ in the case where $G_{2}^{\mathbf{n}}=G_{3}^{\mathbf{n}}=G_{4}^{\mathbf{n}}=0$. Consequently, only four conditions are required at an outflow boundary (cf. Appendix B and [24]). We note that the boundary operator remains well posed when the Reynolds number approaches infinity in which case we recover the characteristic boundary conditions for the inviscid, compressible Euler equations.

4.2. The semidiscrete scheme. Having established a boundary operator ensuring well posedness inside a general volume allows us to develop an asymptotically stable scheme for approximating the compressible Navier-Stokes equations in a curvilinear volume. Although a similar approach may be applied for constructing schemes on general domains, we restrict the attention to the hexahedral domain.

Using a Legendre collocation approximation, we propose to solve the compressible Navier-Stokes equations in a general hexahedral as

$$
\begin{aligned}
\frac{\partial \boldsymbol{q}}{\partial t}+\nabla \cdot \boldsymbol{\Pi}= & \frac{1}{\operatorname{Re}_{\mathrm{ref}}} \nabla \cdot \boldsymbol{\Pi}^{\nu} \\
& -\tau_{i j k} Q(\boldsymbol{x}) \mathcal{S}^{\mathbf{n}}\left[\mathcal{R}_{ \pm}^{\mathbf{n}}\left(\boldsymbol{R}^{\mathbf{n}}-\boldsymbol{R}_{B C}^{\mathbf{n}}\right)+\frac{1}{\operatorname{Re}_{\mathrm{ref}}} \mathcal{G}_{ \pm}\left(\boldsymbol{G}^{\mathbf{n}}-\boldsymbol{G}_{B C}^{\mathbf{n}}\right)\right]
\end{aligned}
$$

where we introduce $\boldsymbol{R}_{B C}^{\mathrm{n}}$ and $\boldsymbol{G}_{B C}^{\mathrm{n}}$ to account for the boundary conditions on characteristic form at the various boundaries, be they subdomain boundaries or open boundaries. The function $Q(\boldsymbol{x})$ is defined in (5) and we recall that $\tau_{i j k} \equiv 0$ for $\boldsymbol{x} \in \mathrm{D} \backslash \delta \mathrm{D}$.

LEMma 4.2. Assume there exists a solution $\boldsymbol{q}$ to the compressible Navier-Stokes equations in a curvilinear hexahedral domain $\mathrm{D}$ enclosed by an almost smooth boundary $\delta \mathrm{D}$. Assume also that there exists an diffeomorphism $\Psi: \mathrm{D} \rightarrow \mathrm{I}$, which maps $\mathrm{D}$ onto the unit cube I.

The fluid properties must by constrained as

$$
\mu \geq 0, \quad \lambda \leq 0, \quad \mu+\lambda \geq 0, \frac{\gamma k}{\operatorname{Pr}} \geq 0, \gamma \geq 1 .
$$

Let

$$
\kappa^{\mathrm{sub}}=\frac{\varepsilon|\boldsymbol{n}|^{2}}{2 \tilde{\omega}} \frac{1}{\rho_{0}\left|\boldsymbol{u}_{0} \cdot \boldsymbol{n}\right|} \max \left(\mu_{0}, 2 \mu_{0}+\lambda_{0}, \frac{\gamma k_{0}}{\mathrm{Pr}}\right)
$$

and

$$
\kappa^{\mathrm{sup}}=\frac{\varepsilon|\boldsymbol{n}|^{2}}{2 \tilde{\omega}} \frac{1}{\rho_{0}\left|\boldsymbol{u}_{0} \cdot \boldsymbol{n}\right|} \max \left(\mu_{0},\left(2 \mu_{0}+\lambda_{0}\right) \frac{\gamma \tilde{M}^{2}}{\gamma \tilde{M}^{2}-1}, \frac{\gamma k_{0}}{\operatorname{Pr}}\right),
$$

where $\tilde{M}=\left|\boldsymbol{u}_{0} \cdot \boldsymbol{n}\right| / c_{0}$ signifies the ratio between the local normal advective velocity and the sound speed.

Approximating the solution of the linearized constant coefficient version of (11) using a Legendre collocation method yields an asymptotically stable scheme provided the penalty parameters are given as follows.

Subsonic inflow/outflow.

$$
\frac{1}{\tilde{\omega} \kappa^{\mathrm{sub}}}\left(1+\kappa^{\mathrm{sub}}-\sqrt{1+\kappa^{\mathrm{sub}}}\right) \leq \tau \leq \frac{1}{\tilde{\omega} \kappa^{\mathrm{sub}}}\left(1+\kappa^{\mathrm{sub}}+\sqrt{1+\kappa^{\mathrm{sub}}}\right) .
$$


Supersonic inflow.

$$
\frac{1}{\tilde{\omega} \kappa^{\text {sup }}}\left(1+\kappa^{\text {sup }}-\sqrt{1+\kappa^{\text {sup }}}\right) \leq \tau \leq \frac{1}{\tilde{\omega} \kappa^{\text {sup }}}\left(1+\kappa^{\text {sup }}+\sqrt{1+\kappa^{\text {sup }}}\right) .
$$

Supersonic outflow.

$$
\frac{1}{\tilde{\omega}}\left(1-\sqrt{\frac{1}{\kappa^{\text {sup }}}}\right) \leq \tau \leq \frac{1}{\tilde{\omega}}\left(1+\sqrt{\frac{1}{\kappa^{\text {sup }}}}\right) .
$$

The correct choices of $\tilde{\omega}$ and the outward pointing normal vector $\boldsymbol{n}$, depending on whether a face, an edge, or a vertex is considered, are given in Appendix A.

For the stable scheme, the growth of the solution is bounded as

$$
\frac{1}{2} \frac{d}{d t}\left\|\mathcal{Q} \boldsymbol{R}^{\mathbf{i}}\right\|_{N}^{2} \leq-\left.\frac{1}{\operatorname{Re}_{\text {ref }}} \sum_{\tilde{\Xi}_{N}}\left(\sum_{m, l=1}^{3}\left(\frac{\partial \boldsymbol{R}^{\mathbf{i}}}{\partial x_{m}}\right)^{T} \mathcal{Q}^{T} \mathcal{Q} \mathcal{B}_{m l}^{\mathbf{i}} \frac{\partial \boldsymbol{R}^{\mathbf{i}}}{\partial x_{l}}\right)\right|_{i j k} J \omega_{i}^{0} \omega_{j}^{0} \omega_{k}^{0} \leq 0 .
$$

Proof. For ease of exposure, we only present the proof for one face, one edge, and one vertex as the remaining parts of the proof follow by a similar approach. Moreover, we only consider supersonic boundary conditions, as the conditions for subsonic boundary conditions are obtained in a similar fashion. It is sufficient to consider homogeneous boundary conditions, i.e., $\boldsymbol{R}_{B C}^{\mathrm{n}}=\boldsymbol{G}_{B C}^{\mathrm{n}}=0$.

To establish the proof, we consider the constant coefficient version of (11), linearized around the uniform solution $\boldsymbol{q}_{0}$, and apply the similarity transform using $\mathcal{S}^{\mathbf{i}}$ and $\left(\mathcal{S}^{\mathbf{i}}\right)^{-1}$. Multiplying with $J \omega_{i}^{0} \omega_{j}^{0} \omega_{k}^{0}\left(\boldsymbol{R}^{\mathbf{i}}\right)^{T} \mathcal{Q}^{T} \mathcal{Q}$ from the left, summing over $\Xi_{N}$, and invoking the quadrature rule and integration by parts, we obtain the energy integral for the hexahedral D as

$$
\begin{aligned}
\frac{1}{2} \frac{d}{d t}\left\|\mathcal{Q} \boldsymbol{R}^{\mathbf{i}}\right\|_{N}^{2}= & \oint_{\delta \mathrm{D}}\left(\boldsymbol{R}^{\mathbf{i}}\right)^{T} \mathcal{Q}^{T} \mathcal{Q}\left(\sum_{i=1}^{3}\left[-\frac{1}{2} \mathcal{A}_{i}^{\mathbf{i}} \boldsymbol{R}^{\mathbf{i}}+\frac{1}{\operatorname{Re}_{\mathrm{ref}}} \sum_{j=1}^{3} \mathcal{B}_{i j}^{\mathbf{i}} \frac{\partial \boldsymbol{R}^{\mathbf{i}}}{\partial x_{j}}\right] n_{i}\right) d s \\
& -\varepsilon \int_{\mathrm{D}} \sum_{i, j=1}^{3}\left(\frac{\partial \boldsymbol{R}^{\mathbf{i}}}{\partial x_{i}}\right)^{T} \mathcal{Q}^{T} \mathcal{Q} \mathcal{B}_{i j}^{\mathbf{i}} \frac{\partial \boldsymbol{R}^{\mathbf{i}}}{\partial x_{j}} d \boldsymbol{x} \\
& -\sum_{\Xi_{N}} \tau_{i j k} Q(\boldsymbol{x})\left(\boldsymbol{R}^{\mathbf{i}}\right)^{T} \mathcal{Q}^{T} \mathcal{Q} \mathcal{H}^{\mathbf{i} \mathbf{n}}\left[\mathcal{R}_{ \pm}^{\mathbf{n}} \boldsymbol{R}^{\mathbf{n}}+\frac{1}{\operatorname{Re}_{\mathrm{ref}}} \mathcal{G}_{ \pm} \boldsymbol{G}^{\mathbf{n}}\right] J \omega_{i}^{0} \omega_{j}^{0} \omega_{k}^{0} \leq 0
\end{aligned}
$$

For notational convenience, we introduce the symbols

$$
\mathcal{A}_{\mathbf{n}}^{\mathbf{i}}=\left(\mathcal{S}^{\mathbf{i}}\right)^{-1} \mathcal{A} \mathcal{S}^{\mathbf{i}}, \quad G_{\mathbf{n}}^{\mathbf{i}}=\left(\mathcal{S}^{\mathbf{i}}\right)^{-1} \mathcal{S}^{\mathbf{n}} G^{\mathbf{n}}=\mathcal{H}^{\mathbf{i}, \mathbf{n}} G^{\mathbf{n}},
$$

with $\mathcal{A}_{\mathbf{n}}^{\mathbf{n}}=\mathcal{A}^{\mathbf{n}}$ being diagonal and $G_{\mathbf{n}}^{\mathbf{n}}=G^{\mathbf{n}}$.

We have $\varepsilon=1 / \operatorname{Re}_{\text {ref }}$ and name the Legendre-Gauss-Lobatto weights at the endpoints as $\omega_{\xi}, \omega_{\eta}$, and $\omega_{\zeta}$ (see Appendix A). We recall the definition of the vectors $\boldsymbol{n}_{\xi}, \boldsymbol{n}_{\eta}$, and $\boldsymbol{n}_{\zeta}$ introduced during the proof of Lemma 3.2. The boundary contribution from the energy integral is, in the special case of a hexahedral, given as

$$
\oint_{\delta \mathrm{D}}\left(\boldsymbol{R}^{\mathbf{i}}\right)^{T} \mathcal{Q}^{T} \mathcal{Q}\left(-\frac{1}{2} \mathcal{A}_{\mathbf{n}}^{\mathbf{i}} \boldsymbol{R}^{\mathbf{i}}+\frac{1}{\operatorname{Re}_{\mathrm{ref}}} \boldsymbol{G}_{\mathbf{n}}^{\mathbf{i}}\right) d s
$$




$$
\begin{aligned}
& =\sum_{\Xi_{N}^{\eta \zeta}}\left[\left(\boldsymbol{R}^{\mathbf{i}}\right)^{T} \mathcal{Q}^{T} \mathcal{Q}\left(-\frac{1}{2} \mathcal{A}_{\mathbf{n}_{\xi}}^{\mathbf{i}} \boldsymbol{R}^{\mathbf{i}}+\frac{1}{\operatorname{Re}_{\mathrm{ref}}} \boldsymbol{G}_{\mathbf{n}_{\xi}}^{\mathbf{i}}\right)\right]_{\xi= \pm 1} J \omega_{j}^{0} \omega_{k}^{0} \\
& +\sum_{\Xi_{N}^{\xi \zeta}}\left[\left(\boldsymbol{R}^{\mathbf{i}}\right)^{T} \mathcal{Q}^{T} \mathcal{Q}\left(-\frac{1}{2} \mathcal{A}_{\mathbf{n}_{\eta}}^{\mathbf{i}} \boldsymbol{R}^{\mathbf{i}}+\frac{1}{\operatorname{Re}_{\mathrm{ref}}} \boldsymbol{G}_{\mathbf{n}_{\eta}}^{\mathbf{i}}\right)\right]_{\eta= \pm 1} J \omega_{i}^{0} \omega_{k}^{0} \\
& +\sum_{\Xi_{N}^{\xi \eta}}\left[\left(\boldsymbol{R}^{\mathbf{i}}\right)^{T} \mathcal{Q}^{T} \mathcal{Q}\left(-\frac{1}{2} \mathcal{A}_{\mathbf{n}_{\zeta}}^{\mathbf{i}} \boldsymbol{R}^{\mathbf{i}}+\frac{1}{\operatorname{Re}_{\mathrm{ref}}} \boldsymbol{G}_{\mathbf{n}_{\zeta}}^{\mathbf{i}}\right)\right]_{\zeta= \pm 1} J \omega_{i}^{0} \omega_{j}^{0} .
\end{aligned}
$$

We now split the treatment into faces, edges and vertices of the hexahedral.

Face: $(\xi, \eta, \zeta)=(-1, \cdot, \cdot)$ and $\boldsymbol{n}=\boldsymbol{n}_{\xi}=-\nabla \xi$. Collecting the terms contributing to stability at the face, excluding all edges and vertices, yields the pointwise condition for stability

$$
\begin{aligned}
\forall(j, k) \in \tilde{\Xi}_{N}^{\eta \zeta}: & \left(\tilde{\boldsymbol{R}}^{\mathbf{i}}\right)^{T} \mathcal{Q}^{T} \mathcal{Q}\left(-\frac{1}{2} \mathcal{A}_{\mathbf{n}}^{\mathbf{i}}-\omega_{\xi} \tau_{0 j k} \mathcal{W}_{ \pm}^{\mathbf{n}}\right) \tilde{\boldsymbol{R}}^{\mathbf{i}} \\
& +\left(\tilde{\boldsymbol{R}}^{\mathbf{i}}\right)^{T} \varepsilon\left(1-\omega_{\xi} \tau_{0 j k}\right) \mathcal{Q}^{T} \mathcal{Q} \tilde{\boldsymbol{G}}_{\mathbf{n}}^{\mathbf{i}} \\
& -\varepsilon \omega_{\xi} \sum_{m, l=1}^{3}\left(\frac{\partial \tilde{\boldsymbol{R}}^{\mathbf{i}}}{\partial x_{m}}\right)^{T} \mathcal{Q}^{T} \mathcal{Q} \mathcal{B}_{m l}^{\mathbf{i}} \frac{\partial \tilde{\boldsymbol{R}}^{\mathbf{i}}}{\partial x_{l}} \leq 0
\end{aligned}
$$

where $\tilde{\boldsymbol{R}}^{\mathbf{n}}=\boldsymbol{R}^{\mathbf{n}}\left(-1, \eta_{j}, \zeta_{k}\right)$ and $\tilde{\boldsymbol{G}}_{\mathbf{n}}^{\mathbf{i}}=\boldsymbol{G}_{\mathbf{n}}^{\mathbf{i}}\left(-1, \eta_{j}, \zeta_{k}\right)$ and we recall that $\mathcal{H}^{\mathbf{i}, \mathbf{n}}=$ $\left(\mathcal{S}^{\mathbf{i}}\right)^{-1} \mathcal{S}^{\mathbf{n}}$. As discussed in [1], assuming that $\mathcal{G}_{ \pm}$equals the identity matrix does not introduce any extra boundary conditions due to the symmetry of $\boldsymbol{G}^{\mathbf{n}}$. We have introduced $\mathcal{W}_{ \pm}^{\mathbf{n}}=\mathcal{H}^{\mathbf{i}, \mathbf{n}} \mathcal{R}_{ \pm}^{\mathbf{n}}\left(\mathcal{H}^{\mathbf{i}, \mathbf{n}}\right)^{-1}$, which for supersonic inflow $\mathcal{W}_{-}^{\mathbf{n}}$ becomes

$$
\mathcal{W}_{-}^{\mathbf{n}}=-\frac{1}{2}\left[\begin{array}{ccccc}
2 \boldsymbol{u}_{0} \cdot \hat{\boldsymbol{n}}+2 c_{0} \hat{n}_{1} & 0 & 2 c_{0} \hat{n}_{3} & -2 c_{0} \hat{n}_{2} & 0 \\
0 & 2 \boldsymbol{u}_{0} \cdot \hat{\boldsymbol{n}} & 0 & 0 & 0 \\
c_{0} \hat{n}_{3} & 0 & 2 \boldsymbol{u}_{0} \cdot \hat{\boldsymbol{n}} & 0 & c_{0} \hat{n}_{3} \\
-c_{0} \hat{n}_{2} & 0 & 0 & 2 \boldsymbol{u}_{0} \cdot \hat{\boldsymbol{n}} & -c_{0} \hat{n}_{2} \\
0 & 0 & 2 c_{0} \hat{n}_{3} & -2 c_{0} \hat{n}_{2} & 2 \boldsymbol{u}_{0} \cdot \hat{\boldsymbol{n}}-2 c_{0} \hat{n}_{1}
\end{array}\right],
$$

while $\mathcal{W}_{+}^{\text {n }}$ equals the null matrix. Consequently, $\mathcal{Q}^{T} \mathcal{Q W}_{ \pm}^{\text {n }}$ is symmetric. The same holds for subsonic conditions although $\mathcal{W}_{ \pm}^{\mathbf{n}}$ is different in this case.

Since all matrices are symmetric, by introducing the characteristic column vector $\boldsymbol{V}=\left(\tilde{\boldsymbol{R}}^{\mathbf{i}}, \nabla \tilde{\boldsymbol{R}}^{\mathbf{i}}\right)^{T}$ we obtain the stability condition on quadratic form $\boldsymbol{V}^{T} \mathcal{Q}^{T} \mathcal{Q} \Pi_{ \pm} \boldsymbol{V} \leq$ 0 , where $\mathcal{Q}^{T} \mathcal{Q} \Pi_{ \pm}$is a 4-by-4 symmetric block matrix. Hence, as for the advectiondiffusion equation, the question of stability can be recast into a symmetric eigenvalue problem similar to what we did for the linear advection-diffusion problem, albeit of significant complexity.

Establishing negative semidefiniteness of a 20 -by-20 symbolic eigenvalue problem is a nontrivial task, and we have found it necessary to employ a significant amount of algebra to obtain the sought-after conditions.

Let us first introduce the primitive variables $\tilde{\boldsymbol{q}}=(\rho, u, v, w, p)^{T}$ and the transformation operator $\mathcal{M}=\partial \tilde{\boldsymbol{q}} / \partial \boldsymbol{q}$, which is the transformation between primitive and conserved variables. The operator is given in [22]. Let us also define the parabolic symmetrizer $\mathcal{S}_{p}$ introduced in [25]. This simultaneously diagonalizes all normal dissipative matrices $\mathcal{B}_{i i}$ while keeping the off-diagonal dissipative matrices very sparse 
and maintaining the symmetry of the convective matrices, provided the problem is stated in primitive variables.

The transformation from the symmetric problem in conserved variables to the primitive variable formulation is accomplished with the operator $\mathcal{L}=\left(\mathcal{S}^{\mathrm{i}}\right)^{-1} \mathcal{M} \mathcal{S}_{p}$ as $\tilde{\Pi}_{ \pm}=\mathcal{L}^{T} \mathcal{Q}^{T} \mathcal{Q} \Pi_{ \pm} \mathcal{L}=\mathcal{L}^{T} \mathcal{Q}^{T} \mathcal{Q} \mathcal{L} \mathcal{L}^{-1} \Pi_{ \pm} \mathcal{L}$. The first part of this expression simplifies since $\mathcal{L}^{T} \mathcal{Q}^{T} \mathcal{Q} \mathcal{L}=2 \rho_{0}^{2} \mathcal{I}$. For the remaining part, $\mathcal{L}$ and $\mathcal{L}^{-1}$ transform and symmetrize all convective and diffusive matrices (see [25] for the explicit entries). To complete the understanding of the problem, we need to address the effect of the transformation on the boundary operator $\mathcal{W}_{ \pm}^{\mathbf{n}}$. We obtain

$$
\tilde{\mathcal{W}}_{ \pm}^{\mathbf{n}}=\mathcal{L}^{-1} \mathcal{W}_{ \pm}^{\mathbf{n}} \mathcal{L}=\mathcal{L}^{-1} \mathcal{H}^{\mathbf{i}, \mathbf{n}} \mathcal{R}_{ \pm}^{\mathbf{n}}\left(\mathcal{H}^{\mathbf{i}, \mathbf{n}}\right)^{-1} \mathcal{L}=\left(\left(\mathcal{S}^{\mathbf{n}}\right)^{-1} \mathcal{M} \mathcal{S}_{p}\right)^{-1} \mathcal{R}_{ \pm}^{\mathbf{n}}\left(\mathcal{S}^{\mathbf{n}}\right)^{-1} \mathcal{M} \mathcal{S}_{p}
$$

We observe that the eigenvalue spectrum $\lambda\left(\tilde{\mathcal{W}}_{ \pm}^{\mathbf{n}}\right)=\lambda\left(\mathcal{R}_{ \pm}^{\mathbf{n}}\right)$ as expected. Computing the entries of $\tilde{\mathcal{W}}_{ \pm}^{\mathbf{n}}$ confirms that it remains symmetric under the transformation, and, consequently, $\tilde{\Pi}_{ \pm}$remains symmetric for supersonic as well as subsonic conditions.

The advantage of this rather involved line of transformations is that all submatrices of $\tilde{\Pi}_{ \pm}$, with the exception of $\left(\tilde{\Pi}_{ \pm}\right)_{11}$, become very sparse. Additionally, the singularity of $\tilde{\Pi}_{ \pm}$, due to the singular nature of the dissipative operator, can be removed by removing the three rows and columns corresponding to mass conservation. The eigenvalue problem is by now nonsingular and block symmetric and we may reduce it to block-diagonal form by repeatedly employing Sylvester's law of inertia for symmetric matrices [23] as we are only concerned about the sign of the eigenvalues. At this point, we have reduced the problem of establishing negative semidefiniteness of a 20-by- 20 matrix to that of three 4 -by- 4 and one 5 -by-5 symmetric matrix being a feasible task. We have used LU-decomposition of the diagonal blocks, and the sought-after conditions appear by requiring the diagonal elements of the upper triangular matrix $\mathrm{U}$ to be nonnegative.

The conditions resulting from the dissipative part of the problem, i.e., from the effects of $\mathcal{B}_{i j}$, remain constant independent of whether an inflow or an outflow point is considered and whether it is subsonic or supersonic. The first 15 eigenvalues $\rho\left(\tilde{\Pi}_{ \pm}\right)$ are found as

$$
\begin{array}{ccc}
\rho_{1}=\rho_{2}=\rho_{3}=0, & \rho_{4}=\rho_{5}=\rho_{6}=-\mu, & \rho_{7}=\rho_{8}=\rho_{9}=-\frac{\gamma k}{\operatorname{Pr}}, \\
\rho_{10}=-(2 \mu+\lambda), & \rho_{11}=-\frac{4(2 \mu+\lambda)^{2}-(\mu+\lambda)^{2}}{4(2 \mu+\lambda)}, \quad \rho_{12}=\rho_{13}=\rho_{14}=-\frac{4 \mu^{2}-(\mu+\lambda)^{2}}{4 \mu}, \\
\rho_{15}=-\frac{2(2 \mu+\lambda)^{2}+(2 \mu+\lambda)(\mu+\lambda)-(\mu+\lambda)^{2}}{2(2 \mu+\lambda)+(\mu+\lambda)} .
\end{array}
$$

It is easily verified that these eigenvalues remain nonpositive under the conditions

$$
\mu \geq 0, \quad \lambda \leq 0, \quad \mu+\lambda \geq 0, \frac{\gamma k}{\operatorname{Pr}} \geq 0,
$$

which are natural thermodynamic relations.

The remaining five eigenvalues are used to ensure stability through conditions on $\tau$. These conditions vary depending on whether an inflow/outflow or a subsonic/supersonic face is considered. For ease of exposure we just give the conditions on $\tau_{i j k}$ leading to stability at a supersonic inflow point as found using the procedure described above. Let us introduce

$$
\tau_{\kappa}^{ \pm}=\frac{1}{\omega_{\xi} \kappa}(1+\kappa \pm \sqrt{1+\kappa})
$$


The conditions for stability are then given as

$$
\begin{array}{ll}
1: & \tau \geq \frac{1}{2 \omega_{\xi}}, \\
2: & \tau_{\kappa}^{+} \geq \tau \geq \tau_{\kappa}^{-}, \quad \kappa=\frac{|\mathbf{n}|^{2} \varepsilon}{2 \omega_{\xi}} \frac{2 \mu+\lambda}{\rho_{0}\left|\mathbf{n} \cdot \mathbf{u}_{0}\right|} \frac{\gamma \tilde{M}^{2}}{\gamma \tilde{M}^{2}-1}, \\
3: & \tau_{\kappa}^{+} \geq \tau \geq \tau_{\kappa}^{-}, \quad \kappa=\frac{|\mathbf{n}|^{2} \varepsilon}{2 \omega_{\xi}} \frac{\mu}{\rho_{0}\left|\mathbf{n} \cdot \mathbf{u}_{0}\right|}, \\
4: & \tau_{\kappa}^{+} \geq \tau \geq \tau_{\kappa}^{-}, \quad \kappa=\frac{|\mathbf{n}|^{2} \varepsilon}{2 \omega_{\xi}} \frac{\mu}{\rho_{0}\left|\mathbf{n} \cdot \mathbf{u}_{0}\right|}, \\
5 \mathrm{a}: & \tau_{\kappa}^{+} \geq \tau \geq \tau_{\kappa}^{-}, \quad \kappa=\frac{|\mathbf{n}|^{2} \varepsilon}{2 \omega_{\xi}} \frac{2 \mu+\lambda}{\rho_{0}\left|\mathbf{n} \cdot \mathbf{u}_{0}\right|} \frac{\gamma \tilde{M}^{2}}{\gamma \tilde{M}^{2}-1}, \\
5 \mathrm{~b}: & \tau_{\kappa}^{+} \geq \tau \geq \tau_{\kappa}^{-}, \quad \kappa=\frac{|\mathbf{n}|^{2} \varepsilon}{2 \omega_{\xi}} \frac{\gamma \kappa}{\operatorname{Pr} \rho_{0}\left|\mathbf{n} \cdot \mathbf{u}_{0}\right|},
\end{array}
$$

where $\tilde{M}=\left|\boldsymbol{u}_{0} \cdot \boldsymbol{n}\right| / c$. This immediately leads to the conditions ensuring stability as stated in the lemma. The remaining three combinations of inflow/outflow conditions are handled in a similar manner, leading to the conditions stated in the lemma. This concludes the part of the proof dealing with a face. Clearly, stability at any other face can be established using the same procedure.

Edge: $(\xi, \eta, \zeta)=(-1,-1, \cdot)$ and $\boldsymbol{n}=\omega_{\eta} \boldsymbol{n}_{\xi}+\omega_{\xi} \boldsymbol{n}_{\eta}$. Collecting the terms contributing to stability along the edge, excluding the vertices, yields the pointwise condition for stability

$$
\begin{aligned}
\forall k \in \tilde{\Xi}_{N}^{\zeta}: & \left(\tilde{\boldsymbol{R}}^{\mathbf{i}}\right)^{T} \mathcal{Q}^{T} \mathcal{Q}\left(-\frac{1}{2} \mathcal{A}_{\mathbf{n}}^{\mathbf{i}}-\tilde{\omega} \tau_{00 k} \mathcal{W}_{ \pm}^{\mathbf{n}}\right) \tilde{\boldsymbol{R}}^{\mathbf{i}} \\
& +\left(\tilde{\boldsymbol{R}}^{\mathbf{i}}\right)^{T} \varepsilon\left(1-\tilde{\omega} \tau_{00 k}\right) \mathcal{Q}^{T} \mathcal{Q} \tilde{\boldsymbol{G}}_{\mathbf{n}}^{\mathbf{i}} \\
& -\varepsilon \tilde{\omega} \sum_{m, l=1}^{3}\left(\frac{\partial \tilde{\boldsymbol{R}}^{\mathbf{i}}}{\partial x_{m}}\right)^{T} \mathcal{Q}^{T} \mathcal{Q} \mathcal{B}_{m l}^{\mathbf{i}} \frac{\partial \tilde{\boldsymbol{R}}^{\mathbf{i}}}{\partial x_{l}} \leq 0
\end{aligned}
$$

where $\tilde{\boldsymbol{R}}^{\mathbf{n}}=\boldsymbol{R}^{\mathbf{n}}\left(-1,-1, \zeta_{k}\right)$ and $\tilde{\boldsymbol{G}}_{\mathbf{n}}^{\mathbf{i}}=\boldsymbol{G}_{\mathbf{n}}^{\mathbf{i}}\left(-1,-1, \zeta_{k}\right)$, and we have introduced $\tilde{\omega}=\omega_{\xi} \omega_{\eta}$. Hence we arrive at an expression equivalent to the one dealt with for the face; i.e., stability is obtained along the edge using a similar expression for $\tau$, with the only difference appearing in the definition of $\boldsymbol{n}$ and $\tilde{\boldsymbol{\omega}}$. The correct choices for these parameters can be found in Appendix A. Stability along the remaining edges can be established in a similar way.

Vertex: $(\xi, \eta, \zeta)=(-1,-1,-1)$ and $\boldsymbol{n}=\omega_{\eta} \omega_{\zeta} \boldsymbol{n}_{\xi}+\omega_{\xi} \omega_{\zeta} \boldsymbol{n}_{\eta}+\omega_{\xi} \omega_{\eta} \boldsymbol{n}_{\zeta}$. Collecting the terms that contribute to stability at the vertex yields the pointwise conditions for stability

$$
\begin{aligned}
\left(\tilde{\boldsymbol{R}}^{\mathbf{i}}\right)^{T} \mathcal{Q}^{T} \mathcal{Q}\left(-\frac{1}{2} \mathcal{A}_{\mathbf{n}}^{\mathbf{i}}-\tilde{\omega} \tau_{000} \mathcal{W}_{ \pm}^{\mathbf{n}}\right) \tilde{\boldsymbol{R}}^{\mathbf{i}} & +\left(\tilde{\boldsymbol{R}}^{\mathbf{i}}\right)^{T} \varepsilon\left(1-\tilde{\omega} \tau_{000}\right) \mathcal{Q}^{T} \mathcal{Q} \tilde{\boldsymbol{G}}_{\mathbf{n}}^{\mathbf{i}} \\
& -\varepsilon \tilde{\omega} \sum_{m, l=1}^{3}\left(\frac{\partial \tilde{\boldsymbol{R}}^{\mathbf{i}}}{\partial x_{m}}\right)^{T} \mathcal{Q}^{T} \mathcal{Q} \mathcal{B}_{m l}^{\mathbf{i}} \frac{\partial \tilde{\boldsymbol{R}}^{\mathbf{i}}}{\partial x_{l}} \leq 0
\end{aligned}
$$

where $\tilde{\boldsymbol{R}}^{\mathbf{n}}=\boldsymbol{R}^{\mathbf{n}}(-1,-1,-1)$ and $\tilde{\boldsymbol{G}}_{\mathbf{n}}^{\mathbf{i}}=\boldsymbol{G}_{\mathbf{n}}^{\mathbf{i}}(-1,-1,-1)$, while we have $\tilde{\omega}=\omega_{\xi} \omega_{\eta} \omega_{\zeta}$. Again, we recover an expression similar to the one studied for the face; i.e., stability is obtained at the vertex by using a similar expression for $\tau$ with the difference appearing in the definition of $\boldsymbol{n}$ and $\tilde{\boldsymbol{\omega}}$. The correct choices for these parameters can be found in Appendix A. Stability at the remaining vertices can be established in a similar fashion. 
The remaining term bounds the growth of the solution as

$$
\frac{1}{2} \frac{d}{d t}\left\|\mathcal{Q} \boldsymbol{R}^{\mathbf{i}}\right\|_{N}^{2} \leq-\left.\frac{1}{\operatorname{Re}_{\mathrm{ref}}} \sum_{\tilde{\Xi}_{N}}\left(\sum_{m, l=1}^{3}\left(\frac{\partial \boldsymbol{R}^{\mathbf{i}}}{\partial x_{m}}\right)^{T} \mathcal{Q}^{T} \mathcal{Q} \mathcal{B}_{m l}^{\mathbf{i}} \frac{\partial \boldsymbol{R}^{\mathbf{i}}}{\partial x_{l}}\right)\right|_{i j k} J \omega_{i}^{0} \omega_{j}^{0} \omega_{k}^{0},
$$

which remains negative.

We note that the proof is strictly valid only for the case when the Jacobian is a constant as commented at the end of 3.1. However, as we shall show shortly, the scheme remains stable also for nonconstant Jacobians, thus establishing a stable method for approximating the compressible Navier-Stokes equations inside a general hexahedral domain.

4.2.1. Stable treatment of solid, no-slip boundaries. The main difference between the solid wall and a general subdomain/open boundary is that the convective velocity $\boldsymbol{u}$ is identically zero at the wall. However, the similarity transform and the stability proof remain valid when treating the solid wall as a subsonic boundary. We have chosen to express the boundary conditions in the state variables $\boldsymbol{q}$, although the treatment is equivalent to requiring reflection of the outgoing characteristic with a correction due to viscous effects.

At a solid wall, we propose the following scheme:

$$
\begin{aligned}
\frac{\partial \boldsymbol{q}}{\partial t}+\nabla \cdot \boldsymbol{\Pi}= & \frac{1}{\operatorname{Re}_{\mathrm{ref}}} \nabla \cdot \boldsymbol{\Pi}^{\nu} \\
& -\tau_{i j k}^{\mathrm{BC}} Q_{\mathrm{BC}}(\boldsymbol{x})\left[\left(\boldsymbol{q}-\boldsymbol{q}_{\mathrm{BC}}\right)+\frac{1}{\operatorname{Re}_{\mathrm{ref}}}\left(\boldsymbol{q}^{\nu}-\boldsymbol{q}_{\mathrm{BC}}^{\nu}\right)\right],
\end{aligned}
$$

where $Q_{\mathrm{BC}}(\boldsymbol{x})=1$ at solid boundaries and zero otherwise. Here we have introduced the viscous boundary operator

$$
\boldsymbol{q}^{\nu}=\frac{1}{\rho}\left[\begin{array}{c}
0 \\
\mu\left(\nabla m_{u} \cdot \hat{\boldsymbol{n}}\right)+\frac{\lambda+\mu}{2}\left(\frac{\partial \mathbf{m}}{\partial x} \cdot \hat{\boldsymbol{n}}+\hat{n}_{1} \nabla \cdot \boldsymbol{m}\right) \\
\mu\left(\nabla m_{v} \cdot \hat{\boldsymbol{n}}\right)+\frac{\lambda+\mu}{2}\left(\frac{\partial \mathbf{m}}{\partial y} \cdot \hat{\boldsymbol{n}}+\hat{n}_{2} \nabla \cdot \boldsymbol{m}\right) \\
\mu\left(\nabla m_{w} \cdot \hat{\boldsymbol{n}}\right)+\frac{\lambda+\mu}{2}\left(\frac{\partial \mathbf{m}}{\partial z} \cdot \hat{\boldsymbol{n}}+\hat{n}_{3} \nabla \cdot \boldsymbol{m}\right) \\
\frac{\gamma k}{\operatorname{Pr}}\left[\nabla E-\frac{E}{\rho} \nabla \rho\right] \cdot \hat{\boldsymbol{n}}
\end{array}\right],
$$

where $\boldsymbol{m}=\left(m_{u}, m_{v}, m_{w}\right)=\rho(u, v, w)$ signifies the momentum and $\hat{\boldsymbol{n}}$ represents an outward pointing unit vector at the wall. The boundary conditions, imposed though the two vectors $\boldsymbol{q}_{\mathrm{BC}}$ and $\boldsymbol{q}_{\mathrm{BC}}^{\nu}$, depend on the specific problem being considered.

For isothermal boundary conditions, with the boundary being held at the temperature $T_{\mathrm{BC}}$, we use

$$
\boldsymbol{q}_{\mathrm{BC}}=\left[\begin{array}{c}
\rho \\
0 \\
0 \\
0 \\
\rho T_{\mathrm{BC}}
\end{array}\right], \boldsymbol{q}_{\mathrm{BC}}^{\nu}=\left[\begin{array}{c}
0 \\
\hat{n}_{1} \tau_{x x}+\hat{n}_{2} \tau_{x y}+\hat{n}_{3} \tau_{x z} \\
\hat{n}_{1} \tau_{y x}+\hat{n}_{2} \tau_{y y}+\hat{n}_{3} \tau_{y z} \\
\hat{n}_{1} \tau_{z x}+\hat{n}_{2} \tau_{z y}+\hat{n}_{3} \tau_{z z} \\
\frac{\gamma k}{\mathrm{Pr}} \nabla T \cdot \hat{\boldsymbol{n}}
\end{array}\right]
$$

Here $\tau_{x_{i} x_{j}}$ represents the stress-tensor elements as given in (10). These boundary conditions ensure reflection of the outward propagating inviscid characteristic and 


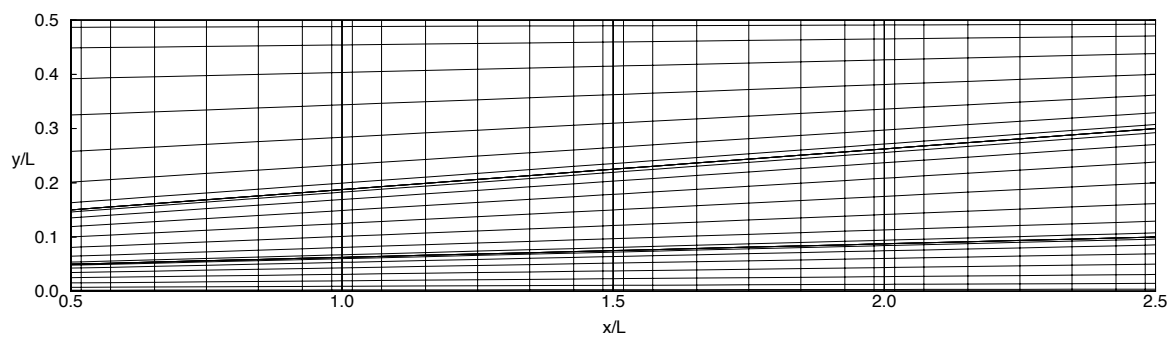

FIG. 6. Example of 12 domain grid with $N_{\xi}=N_{\eta}=8$ used for compressible boundary layer computations.

enforce zero stress along the boundary, while leaving the density, the normal stress, and the temperature gradient to be determined by the computation.

For adiabatic boundary conditions we advocate using

$$
\boldsymbol{q}_{\mathrm{BC}}=\left[\begin{array}{c}
\rho \\
0 \\
0 \\
0 \\
E
\end{array}\right], \quad \boldsymbol{q}_{\mathrm{BC}}^{\nu}=\left[\begin{array}{c}
0 \\
\hat{n}_{1} \tau_{x x}+\hat{n}_{2} \tau_{x y}+\hat{n}_{3} \tau_{x z} \\
\hat{n}_{1} \tau_{y x}+\hat{n}_{2} \tau_{y y}+\hat{n}_{3} \tau_{y z} \\
\hat{n}_{1} \tau_{z x}+\hat{n}_{2} \tau_{z y}+\hat{n}_{3} \tau_{z z} \\
0
\end{array}\right],
$$

thereby enforcing a zero normal temperature gradient at the solid boundary.

The stability for both schemes is straightforwardly established by using the stability condition for a subsonic inflow boundary with vanishing convective velocity, leading to a penalty parameter ensuring stability as

$$
\tau_{i j k}^{\mathrm{BC}}=\frac{|\boldsymbol{n}|}{\tilde{\omega}},
$$

where the actual definition of $\boldsymbol{n}$ and $\tilde{\boldsymbol{\omega}}$ depends on the location of the solid boundary point as discussed in Appendix A.

4.3. Applications. In the previous sections we established asymptotic stability of (11) for the linearized compressible Navier-Stokes equation within a single hexahedral with a constant Jacobian. To verify that all results carry over to the multidomain nonlinear case with general Jacobians, we have implemented a two-dimensional version of the scheme using the curvilinear quadrilateral as the fundamental building block. Since the characteristic vectors $\boldsymbol{R}^{\mathbf{n}}$ and $\boldsymbol{G}^{\mathbf{n}}$ required for the patching of the twodimensional subdomains do not follow straightforwardly from the three-dimensional case, we have included the necessary results in Appendix C. Although the theoretical results were obtained using a Legendre collocation method, we have chosen to use a Chebyshev method to illustrate that the stability results carry over to this important case. The bounds on $\tau_{i j}$ remain unchanged as does the remaining part of the scheme. In particular, vertices are treated as discussed in 3.1.1.

As a viable test case we have chosen a multidomain solution of compressible boundary layer flows over a flat plate, for which we can obtain an accurate solution by solving the boundary layer equations. For this purpose, we use a spectral boundary layer code [26]. We use the multidomain grid shown in Fig. 6 in all computations, albeit with different polynomial order in the domains depending on the actual problem. 
a)

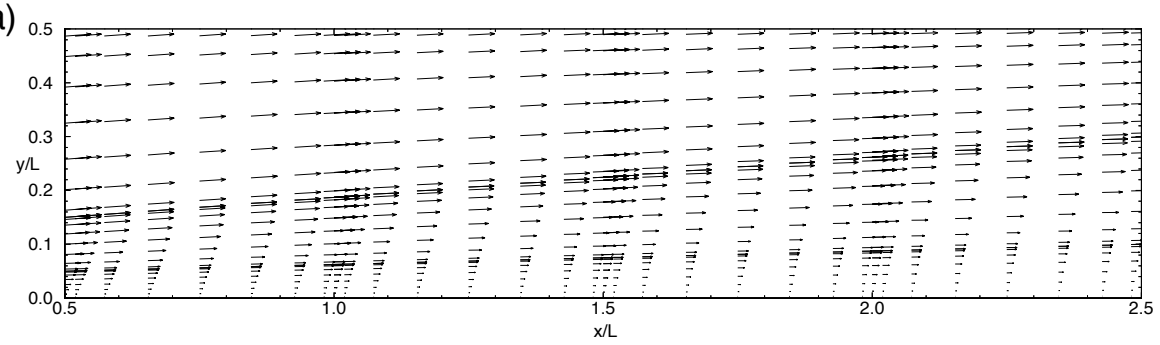

b)

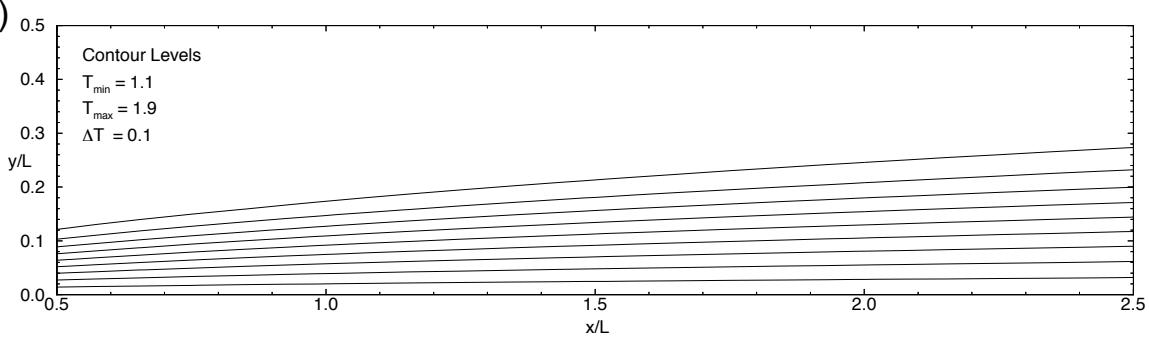

FIG. 7. Steady-state solution of a flat plate subsonic isothermal flow with $R e=1000, M=0.75$, and temperature $T_{\infty}=300^{\circ} \mathrm{K}$ as computed on the grid shown in Fig. 6 with $N_{\xi}=N_{\eta}=8$. (a) Velocity field, $\mathbf{u} / u_{\infty}$. (b) Temperature field, $T / T_{\infty}$.

Boundary conditions for the simulations are obtained from the spectral boundary layer code, which also supplies the wall-normal derivatives at the inflow and outflow boundaries, and we assume that the streamwise variation is sufficiently small to be neglected. The upper boundary in assumed to be in a uniform flow and, thus, all derivatives are assumed to vanish.

Although we are dealing with a steady-state problem, the problems are being solved as if they were fully unsteady. For temporal integration, we have used a third-order Runge-Kutta method with the boundary conditions being imposed at the intermediate time-steps. Following completion of each time-step, we enforce global continuity and use the solution at the previous time-step as the solution around which we linearize at the subdomain boundaries, while the exact solution is used at the open boundaries. The time-step is computed adaptively as

$$
\Delta t \leq \mathrm{CFL} \times \min _{\mathbf{x} \in \Omega}\left[|\chi \cdot \boldsymbol{u}|+c \sqrt{\chi \cdot \chi}+\frac{2 \gamma}{\operatorname{PrRe}_{\mathrm{ref}}} \frac{\mu}{\rho} \chi \cdot \chi\right]^{-1},
$$

where $\chi$ is the grid-distortion measure given in (8).

As advocated in the previous papers of this trilogy $[1,2]$, we choose the minimum penalty parameter; i.e., $\tau_{i j k}=\tau_{i j k}^{-}$from Lemma 4.2. Moreover, we reduce the penalty parameters relative to the parameters resulting from the theoretical analysis by a factor of 2. A thorough discussion of this scaling can be found in [1]. Only at the solid boundaries do we use the value resulting from the theoretical analysis. We use $\mathrm{CFL} \simeq 0.5-1$ in all simulations, i.e., a reduction as compared with the results reported in [2]. However, contrary to the schemes presented there, we do not use any filters in the present paper. Consequently, we could increase the CFL number by using filters in the implementation, albeit at the expense of a slight loss of resolution power. We have chosen to leave the choice of whether to use filters or not to the reader. 

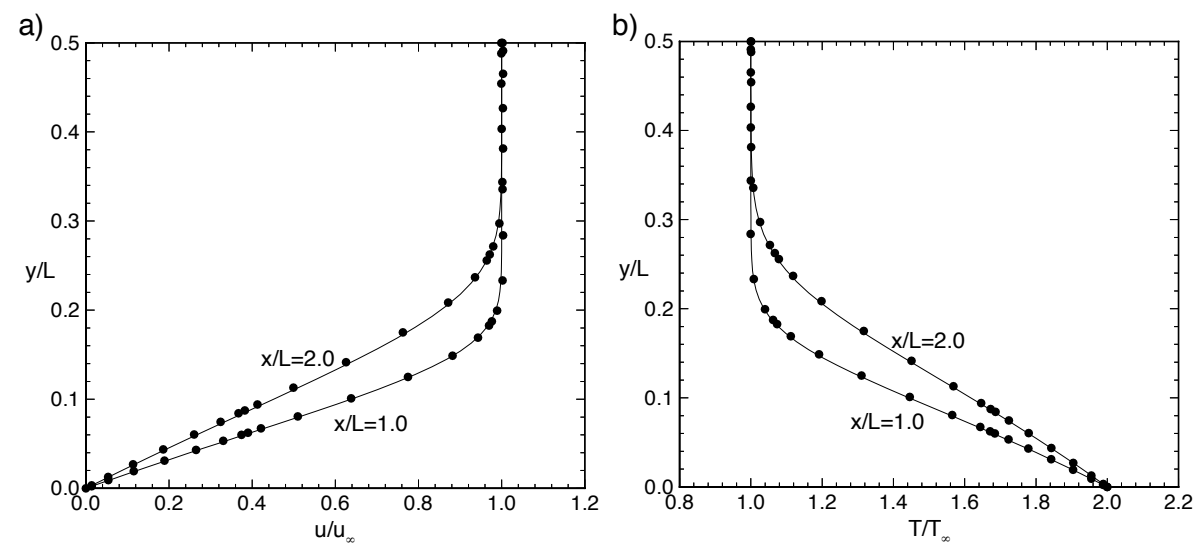

FIG. 8. Steady-state profiles of a flat plate subsonic isothermal flow with Re $=1000, M=0.75$, and temperature $T_{\infty}=300^{\circ} \mathrm{K}$ as obtained by solving the boundary layer equations (solid line) and by using the multidomain scheme (solid circles). (a) Velocity profile $u / u_{\infty}$. (b) Temperature profile $T / T_{\infty}$.

4.3.1. Subsonic isothermal flow over a flat plate. As a first test case, we consider the steady-state solution of a subsonic flow over an isothermal flat plate. The computation is performed in the domain $\Omega=[0.5,2.5] \times[0.0,0.5]$, which is split into 12 quadrilaterals as illustrated in Fig. 6 . We use isotropic resolution with $N_{\xi}=N_{\eta}=8$ in all subdomains and choose $\operatorname{Re}=1000, M=0.75$, and $T_{\infty}=300^{\circ} \mathrm{K}$ as the flow parameters. The Reynolds number is based on the free-stream flow and a unit length; i.e., $L=1.0 \mathrm{~m}$. The solid wall is assumed isothermal at a temperature of $T_{\text {wall }}=2 T_{\infty}$. All results are obtained with an $L^{\infty}$-residual of the order of the machine accuracy.

In Fig. 7 we show the computed velocity field $\boldsymbol{u} / u_{\infty}$ and the normalized temperature field $T / T_{\infty}$. We observe that all variables are continuous and smooth across subdomain boundaries, including the vertices.

To verify the scheme quantitatively, we compare in Fig. 8 the streamwise velocity profile $u / u_{\infty}$ and the temperature profile $T / T_{\infty}$ as computed and compared with those obtained by solving the boundary layer equations spectrally.

The two results are found to agree well. The slight difference between the results is caused by a lack of accurate information about the gradients of the flow variables at the open boundaries. However, at $x / L=2.0$ the error in the computed free-stream velocity is at most $0.3 \%$, the error in the free-stream temperature is at most $0.1 \%$, while the maximum variation in the otherwise constant pressure is $0.3 \%$, confirming the accuracy of the scheme. This level of accuracy compares well with that reported in [9] for a similar problem.

4.3.2. Supersonic adiabatic flow over a flat plate. As a second test case we choose to consider supersonic flow over an adiabatic flat plate. The computation is again performed in the domain $\Omega=[0.5,2.5] \times[0.0,0.5]$, which is split into 12 quadrilaterals; see Fig. 6 . We use an isotropic resolution with $N_{\xi}=N_{\eta}=12$ in all subdomains and we choose $\operatorname{Re}=2500, M=2.50$, and $T_{\infty}=300^{\circ} \mathrm{K}$. The Reynolds number is based on the free-stream flow and a unit length; i.e., $L=1.0 \mathrm{~m}$. All results are obtained with an $L^{\infty}$-residual of the order of the machine accuracy. In Fig. 9 we illustrate the computed velocity field $\boldsymbol{u} / u_{\infty}$ and the normalized temperature field $T / T_{\infty}$. Similar to the previous test case, we find that all variables are continuous and 
a)

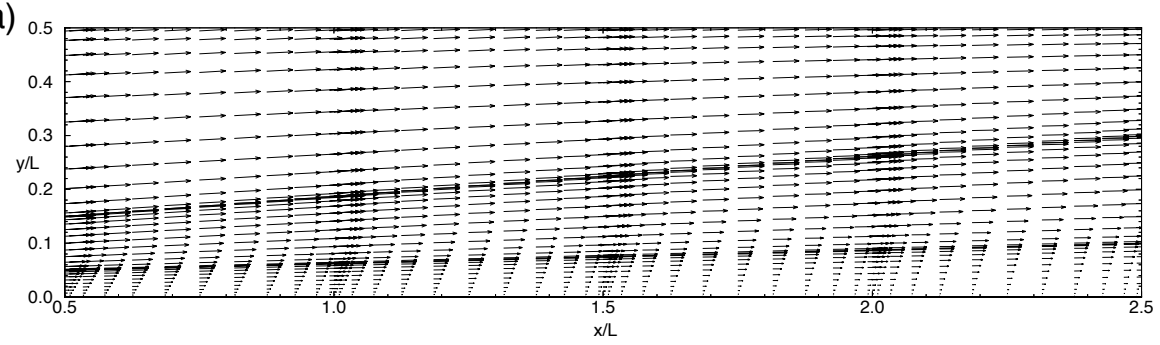

b)

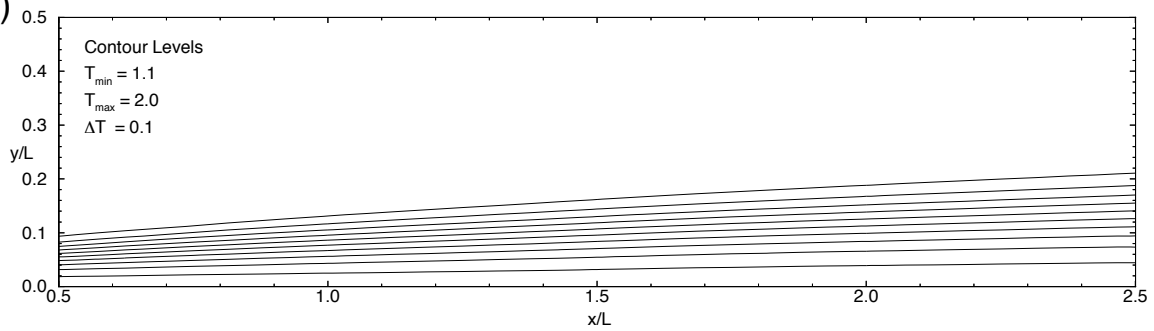

FIG. 9. Steady-state solution of a flat plate supersonic adiabatic flow with $R e=2500, M=2.5$, and temperature $T_{\infty}=300^{\circ} \mathrm{K}$ as computed on the grid shown in Fig. 6 with $N_{\xi}=N_{\eta}=12$. (a) Velocity field $\mathbf{u} / u_{\infty}$. (b) Temperature field $T / T_{\infty}$.

a)

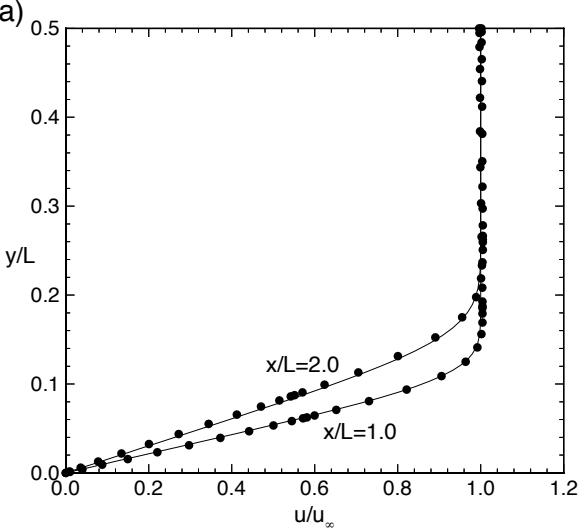

b)

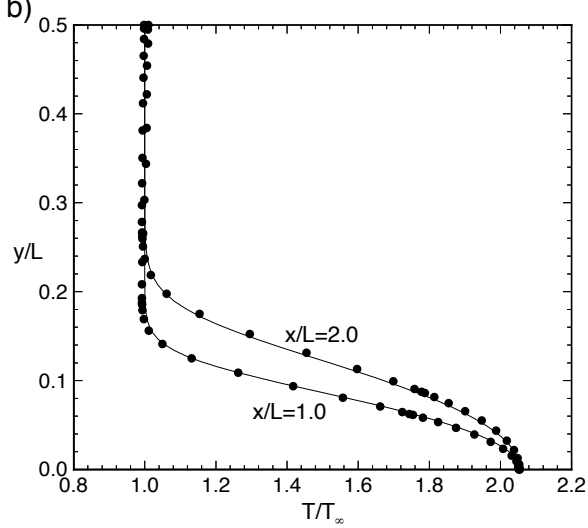

Fig. 10. Steady-state profiles of a flat plate supersonic adiabatic flow with $R e=2500, M=2.5$, and temperature $T_{\infty}=300^{\circ} \mathrm{K}$ as obtained by solving the boundary layer equations (solid line) and by using the multidomain scheme (solid circles). (a) Velocity profile $u / u_{\infty}$. (b) Temperature profile $T / T_{\infty}$

smooth across subdomain boundaries, including the vertices.

In Fig. 10 we compare the computed streamwise velocity $u / u_{\infty}$ and temperature $T / T_{\infty}$, profiles with those obtained by solving the boundary layer equations. Again we observe good agreement. At $x / L=2.0$ the error in the free-stream velocity is at most $0.4 \%$ and the error in the free-stream temperature is at most $0.2 \%$, which is similar to what was reported in [9] for a similar test case.

5. Concluding remarks. This paper completes the ab initio development, initiated in the two previous papers $[1,2]$, of a scheme for the multidomain solution 
of the unsteady three-dimensional compressible Navier-Stokes equations. We have succeeded in developing an asymptotically stable scheme for approximating the compressible Navier-Stokes in a general curvilinear hexahedral. The discussion of this trilogy includes stable treatment of several types of physical boundaries, e.g., open boundaries and isothermal/adiabatic solid boundaries, as well as subdomain boundaries, all approaches sharing the property that the schemes remain stable in the limit of vanishing viscosity, thus being valid also for the compressible Euler equations.

We have only presented numerical results for the two-dimensional case. However, the full theoretical framework has been developed for the three-dimensional problem in curvilinear coordinates, and the success of the latter follows immediately from that of the former as the two schemes are equivalent.

Although we have not addressed the question of efficient implementation, we note that since the penalty treatment of the subdomain and physical boundaries is purely local in time and space, the algorithm promises to allow for an efficient implementation on parallel computers with distributed memory [27]. However, it is premature to claim that an efficient parallel algorithm will result from an implementation and more studies are required to answer this question with certainty. On the other hand, introducing adaptivity by varying the order of the polynomials in each subdomain does not influence the stability of the scheme as stability is established individually in each subdomain (see also [2]). Preliminary studies show that a wavelet decomposition of one or several elements of the state vector yields a good measure of the maximum error in each subdomain, thereby supplying the required local error estimator. This work is currently in progress, and the results shall be reported in a future paper.

We would like to conclude this trilogy with a few general comments regarding the theoretical framework developed here. The success of the development of the schemes hinges on the fact that the convective as well as the diffusive matrices of the compressible Navier-Stokes equations symmetrize simultaneously. This observation is the single most important property required for the development of the stable schemes. We chose to develop the scheme for the conservation form of the Navier-Stokes equations, thereby being forced to apply linearization and localization in order to arrive at the symmetric form. However, an alternative scheme could be derived for the truly nonlinear Navier-Stokes equations by introducing the symmetrizing entropy-flux pair suggested in [28]. This results in a nonlinear symmetric form of the equations and, consequently, allows for the development of a scheme similar to the one presented here. Whether such a scheme is superior to the present one remains unknown. However, using the nonlinear symmetric form relaxes the requirement for smooth solutions which may eventually result in better schemes for high Reynolds- and Mach-number flows.

Appendix A: Geometry and resolution dependent parameters for the specification of the penalty parameters. Let us first define the vectors

$$
\boldsymbol{n}_{\xi}=\xi \nabla \xi, \boldsymbol{n}_{\eta}=\eta \nabla \eta, \boldsymbol{n}_{\zeta}=\zeta \nabla \zeta
$$

We will also introduce the three variables, $\omega_{\xi}, \omega_{\eta}$, and $\omega_{\zeta}$. The actual value of these parameters are resolution as well as method dependent. 
For Legendre methods, we have

$$
\omega_{\xi}=\frac{2}{N_{\xi}\left(N_{\xi}+1\right)}, \quad \omega_{\eta}=\frac{2}{N_{\eta}\left(N_{\eta}+1\right)}, \quad \omega_{\zeta}=\frac{2}{N_{\zeta}\left(N_{\zeta}+1\right)},
$$

where $N_{\xi}, N_{\eta}$, and $N_{\zeta}$ represent the resolution along $\xi, \eta$, and $\zeta$ respectively.

For Chebyshev methods, we have

$$
\omega_{\xi}=\frac{1}{N_{\xi}^{2}}, \quad \omega_{\eta}=\frac{1}{N_{\eta}^{2}}, \quad \omega_{\zeta}=\frac{1}{N_{\zeta}^{2}} .
$$

The appropriate values of the parameter $\tilde{\omega}$ and the outward pointing normal vector $\boldsymbol{n}$ required to construct stable schemes along faces, edges, and vertices of the hexahedral is given below, where we also give the condition for determining whether an edge/vertex is a principal outflow point. For this purpose, we introduce the convective velocity $\boldsymbol{u}$. The conditions for a principal inflow is obtained by reversing the inequalities.

\begin{tabular}{||c|c|c||c|c||c|c|c||}
\hline \multicolumn{2}{||c|}{} & \multicolumn{3}{c||}{$\tau$ - Parameters } & \multicolumn{3}{c||}{ Outflow conditions } \\
\hline$\xi$ & $\eta$ & $\zeta$ & $\tilde{\omega}$ & $\boldsymbol{n}$ & $\boldsymbol{u} \cdot \boldsymbol{n}_{\xi}$ & $\boldsymbol{u} \cdot \boldsymbol{n}_{\eta}$ & $\boldsymbol{u} \cdot \boldsymbol{n}_{\zeta}$ \\
\hline \pm 1 & $\cdot$ & $\cdot$ & $\omega_{\xi}$ & $\boldsymbol{n}_{\xi}$ & $>0$ & - & - \\
$\cdot$ & \pm 1 & $\cdot$ & $\omega_{\eta}$ & $\boldsymbol{n}_{\eta}$ & - & $>0$ & - \\
$\cdot$ & $\cdot$ & \pm 1 & $\omega_{\zeta}$ & $\boldsymbol{n}_{\zeta}$ & - & - & $>0$ \\
\hline \pm 1 & \pm 1 & $\cdot$ & $\omega_{\xi} \omega_{\eta}$ & $\omega_{\eta} \boldsymbol{n}_{\xi}+\omega_{\xi} \boldsymbol{n}_{\eta}$ & $>0$ & $>0$ & - \\
\pm 1 & $\cdot$ & \pm 1 & $\omega_{\xi} \omega_{\zeta}$ & $\omega_{\zeta} \boldsymbol{n}_{\xi}+\omega_{\xi} \boldsymbol{n}_{\zeta}$ & $>0$ & - & $>0$ \\
$\cdot$ & \pm 1 & \pm 1 & $\omega_{\eta} \omega_{\zeta}$ & $\omega_{\zeta} \boldsymbol{n}_{\eta}+\omega_{\eta} \boldsymbol{n}_{\zeta}$ & - & $>0$ & $>0$ \\
\hline \pm 1 & \pm 1 & \pm 1 & $\omega_{\xi} \omega_{\eta} \omega_{\zeta}$ & $\omega_{\eta} \omega_{\zeta} \boldsymbol{n}_{\xi}+\omega_{\xi} \omega_{\zeta} \boldsymbol{n}_{\eta}+\omega_{\xi} \omega_{\eta} \boldsymbol{n}_{\zeta}$ & $>0$ & $>0$ & $>0$ \\
\hline
\end{tabular}

Appendix B: Characteristic vectors and the similarity transform for the three-dimensional compressible Navier-Stokes equations. The similarity transform utilized in section 4 to symmetrize the Navier-Stokes equations along the normal unit vector $\hat{\boldsymbol{n}}=\left(\hat{n}_{1}, \hat{n}_{2}, \hat{n}_{3}\right)$ is

$$
\mathcal{S}^{\mathbf{n}}=\left[\begin{array}{cccc}
\alpha & \frac{\hat{n}_{1}}{\rho} & \frac{\hat{n}_{2}}{\rho} & \\
\alpha\left(u+c \hat{n}_{1}\right) & \hat{n}_{1} \frac{u}{\rho} & -\hat{n}_{3}+\hat{n}_{2} \frac{u}{\rho} & \\
\alpha\left(v+c \hat{n}_{2}\right) & \hat{n}_{3}+\hat{n}_{1} \frac{v}{\rho} & \hat{n}_{2} \frac{v}{\rho} & \\
\alpha\left(w+c \hat{n}_{3}\right) & -\hat{n}_{2}+\hat{n}_{1} \frac{w}{\rho} & \hat{n}_{1}+\hat{n}_{2} \frac{w}{\rho} & \\
\alpha(H+c \boldsymbol{u} \cdot \hat{\boldsymbol{n}}) & (\boldsymbol{u} \times \hat{\boldsymbol{n}}) \cdot \hat{\boldsymbol{\imath}}+\frac{\hat{n}_{1}}{2 \rho} q^{2} & (\boldsymbol{u} \times \hat{\boldsymbol{n}}) \cdot \hat{\boldsymbol{\jmath}}+\frac{\hat{n}_{2}}{2 \rho} q^{2} & \\
& \frac{\hat{n}_{3}}{\rho} & \alpha \\
& \hat{n}_{2}+\hat{n}_{3} \frac{u}{\rho} & \alpha\left(u-c \hat{n}_{1}\right) \\
& -\hat{n}_{1}+\hat{n}_{3} \frac{v}{\rho} & \alpha\left(v-c \hat{n}_{2}\right) \\
& \hat{n}_{3} \frac{w}{\rho} & \alpha\left(w-c \hat{n}_{3}\right) \\
& (\boldsymbol{u} \times \hat{\boldsymbol{n}}) \cdot \boldsymbol{k}+\frac{\hat{n}_{3}}{2 \rho} q^{2} & \alpha(H-c \boldsymbol{u} \cdot \hat{\boldsymbol{n}})
\end{array}\right] .
$$

Here $\boldsymbol{u}=(u, v, w)$ is the convective velocity vector and $q^{2}=\boldsymbol{u} \cdot \boldsymbol{u}$. The Cartesian coordinate system is spanned by the vectors $(\hat{\boldsymbol{\imath}}, \hat{\boldsymbol{\jmath}}, \hat{\boldsymbol{k}})$, and we have introduced the constant $\alpha=1 /(2 c)$ and the specific stagnation enthalpy $H=(E+p) / \rho$.

The inverse of the transformation matrix is given as 


$$
\left(\mathcal{S}^{\mathbf{n}}\right)^{-1}=\left[\begin{array}{ccc}
\beta\left(\frac{1}{2} q^{2}-c \frac{\boldsymbol{u} \cdot \hat{\boldsymbol{n}}}{\gamma-1}\right) & -\beta\left(u-\frac{c \hat{n}_{1}}{\gamma-1}\right) & -\beta\left(v-\frac{c \hat{n}_{2}}{\gamma-1}\right) \\
\rho \hat{n}_{1}\left(1-\frac{\tilde{\beta}}{2 \rho} q^{2}\right)-(\boldsymbol{u} \times \hat{\boldsymbol{n}}) \cdot \hat{\boldsymbol{\imath}} & \tilde{\beta} u \hat{n}_{1} & \hat{n}_{3}+\tilde{\beta} v \hat{n}_{1} \\
\rho \hat{n}_{2}\left(1-\frac{\tilde{\beta}}{2 \rho} q^{2}\right)-(\boldsymbol{u} \times \hat{\boldsymbol{n}}) \cdot \hat{\boldsymbol{\jmath}} & -\hat{n}_{3}+\tilde{\beta} u \hat{n}_{2} & \tilde{\beta} v \hat{n}_{2} \\
\rho \hat{n}_{3}\left(1-\frac{\tilde{\beta}}{2 \rho} q^{2}\right)-(\boldsymbol{u} \times \hat{\boldsymbol{n}}) \cdot \hat{\boldsymbol{k}} & \hat{n}_{2}+\tilde{\beta} u \hat{n}_{3} & -\hat{n}_{1}+\tilde{\beta} v \hat{n}_{3} \\
\beta\left(\frac{1}{2} q^{2}+c \frac{\boldsymbol{u} \cdot \hat{\boldsymbol{n}}}{\gamma-1}\right) & -\beta\left(u+\frac{c \hat{n}_{1}}{\gamma-1}\right) & -\beta\left(v+\frac{c \hat{n}_{2}}{\gamma-1}\right) \\
& -\beta\left(w-\frac{c \hat{n}_{3}}{\gamma-1}\right) & \beta \\
-\tilde{\beta} \hat{n}_{2}+\tilde{\beta} w \hat{n}_{1} & -\tilde{\beta} \hat{n}_{1} \\
& \hat{n}_{1}+\tilde{\beta} w \hat{n}_{2} & -\tilde{\beta} \hat{n}_{2} \\
\tilde{\beta} w \hat{n}_{3} & -\tilde{\beta} \hat{n}_{3} \\
-\beta\left(w+\frac{c \hat{n}_{3}}{\gamma-1}\right) & \beta
\end{array}\right],
$$

where $\beta=(\gamma-1) / c$ and $\tilde{\beta}=(\gamma-1) /\left(c^{2} \rho\right)$. In $\left(\mathcal{S}^{\mathbf{n}}\right)^{-1}$ and $\mathcal{S}^{\mathbf{n}}$ all physical variables refer to the state $\boldsymbol{q}_{0}$ around which we have linearized. Using the similarity transform, we obtain the characteristic variables $\boldsymbol{R}^{\mathbf{n}}=\left(\mathcal{S}^{\mathbf{n}}\right)^{-1} \boldsymbol{q}$ as

$$
\boldsymbol{R}^{\mathbf{n}}=\left[\begin{array}{c}
\boldsymbol{m} \cdot \hat{\boldsymbol{n}}+\frac{\gamma-1}{c_{0}}\left(E+\frac{1}{2} \rho q_{0}^{2}-\rho \boldsymbol{u}_{0} \cdot \boldsymbol{u}\right) \\
\hat{n}_{1} \rho_{0}\left[\rho-\frac{\gamma-1}{c_{0}^{2}}\left(E+\frac{1}{2} \rho q_{0}^{2}-\rho \boldsymbol{u}_{0} \cdot \boldsymbol{u}\right)\right]+(\boldsymbol{m} \times \hat{\boldsymbol{n}}) \cdot \hat{\boldsymbol{\imath}} \\
\hat{n}_{2} \rho_{0}\left[\rho-\frac{\gamma-1}{c_{0}^{2}}\left(E+\frac{1}{2} \rho q_{0}^{2}-\rho \boldsymbol{u}_{0} \cdot \boldsymbol{u}\right)\right]+(\boldsymbol{m} \times \hat{\boldsymbol{n}}) \cdot \hat{\boldsymbol{\jmath}} \\
\hat{n}_{3} \rho_{0}\left[\rho-\frac{\gamma-1}{c_{0}^{2}}\left(E+\frac{1}{2} \rho q_{0}^{2}-\rho \boldsymbol{u}_{0} \cdot \boldsymbol{u}\right)\right]+(\boldsymbol{m} \times \hat{\boldsymbol{n}}) \cdot \hat{\boldsymbol{k}} \\
-\boldsymbol{m} \cdot \hat{\boldsymbol{n}}+\frac{\gamma-1}{c_{0}}\left(E+\frac{1}{2} \rho q_{0}^{2}-\rho \boldsymbol{u}_{0} \cdot \boldsymbol{u}\right)
\end{array}\right],
$$

where we have the linearized momentum $\boldsymbol{m}=\rho\left(\boldsymbol{u}-\boldsymbol{u}_{0}\right)$, and all subscripts refer to the values of the linearized state $\boldsymbol{q}_{0}$.

In deriving the proper well-posed boundary operator, we introduced the viscous correction vector with the explicit entries being

$$
\boldsymbol{G}^{\mathbf{n}}=\left[\begin{array}{c}
\frac{k_{0}(\gamma-1)}{2 \operatorname{Pr} \rho_{0}} \boldsymbol{V}_{1}+\frac{\lambda_{0}+2 \mu_{0}}{2 \rho_{0}} \boldsymbol{V}_{3}-\frac{\lambda_{0}+\mu_{0}}{2 \rho_{0}} \boldsymbol{V}_{4} \\
-\hat{n}_{1} \frac{k_{0}(\gamma-1)}{2 \operatorname{Prc} c_{0}} \boldsymbol{V}_{1}+\frac{\mu_{0}}{\rho_{0}}\left(\nabla R_{2}^{\mathbf{n}}-\hat{n}_{1} \boldsymbol{V}_{2}\right)-\frac{\lambda_{0}+\mu_{0}}{4 \rho_{0}}\left(\boldsymbol{V}_{3} \times \hat{\boldsymbol{\imath}}\right) \\
-\hat{n}_{2} \frac{k_{0}(\gamma-1)}{2 \operatorname{Prc} c_{0}} \boldsymbol{V}_{1}+\frac{\mu_{0}}{\rho_{0}}\left(\nabla R_{3}^{\mathbf{n}}-\hat{n}_{2} \boldsymbol{V}_{2}\right)-\frac{\lambda_{0}+\mu_{0}}{4 \rho_{0}}\left(\boldsymbol{V}_{3} \times \hat{\boldsymbol{\jmath}}\right) \\
-\hat{n}_{3} \frac{k_{0}(\gamma-1)}{2 \operatorname{Prc} c_{0}} \boldsymbol{V}_{1}+\frac{\mu_{0}}{\rho_{0}}\left(\nabla R_{4}^{\mathbf{n}}-\hat{n}_{3} \boldsymbol{V}_{2}\right)-\frac{\lambda_{0}+\mu_{0}}{4 \rho_{0}}\left(\boldsymbol{V}_{3} \times \hat{\boldsymbol{k}}\right) \\
\frac{k_{0}(\gamma-1)}{2 \operatorname{Pr} \rho_{0}} \boldsymbol{V}_{1}-\frac{\lambda_{0}+2 \mu_{0}}{2 \rho_{0}} \boldsymbol{V}_{3}+\frac{\lambda_{0}+\mu_{0}}{2 \rho_{0}} \boldsymbol{V}_{4}
\end{array}\right] \cdot \boldsymbol{n}
$$

where we, for simplicity, introduce the vectors

$$
\begin{gathered}
\boldsymbol{V}_{1}=\nabla R_{1}^{\mathbf{n}}+\nabla R_{5}^{\mathbf{n}}-\frac{2 c_{0}}{\rho_{0}(\gamma-1)} \boldsymbol{V}_{2}, \quad \boldsymbol{V}_{2}=\hat{n}_{1} \nabla R_{2}^{\mathbf{n}}+\hat{n}_{2} \nabla R_{3}^{\mathbf{n}}+\hat{n}_{3} \nabla R_{4}^{\mathbf{n}}, \\
\boldsymbol{V}_{3}=\nabla R_{1}^{\mathbf{n}}-\nabla R_{5}^{\mathbf{n}}, \quad \boldsymbol{V}_{4}=\nabla \times\left(R_{2}^{\mathbf{n}}, R_{3}^{\mathbf{n}}, R_{4}^{\mathbf{n}}\right) .
\end{gathered}
$$

A physical interpretation of these vectors can be found in $[1,2]$. We note the following relationship between the components of the viscous correction vector

$$
\hat{n}_{1} G_{2}^{\mathbf{n}}+\hat{n}_{2} G_{3}^{\mathbf{n}}+\hat{n}_{3} G_{4}^{\mathbf{n}}=-\frac{k_{0}(\gamma-1)}{2 \operatorname{Pr} c_{0}} \boldsymbol{V}_{1} \cdot \boldsymbol{n} .
$$


Appendix C: Characteristic vectors and the similarity transform for the two-dimensional compressible Navier-Stokes equations. As the characteristic vectors for the two-dimensional scheme do not appear directly from the three-dimensional formulation, we give them here for reference. The formulation is valid along the vector $\boldsymbol{n}=|\boldsymbol{n}| \hat{\boldsymbol{n}}=|\boldsymbol{n}|\left(\hat{n}_{1}, \hat{n}_{2}\right)$. For convenience, we also define the tangential vector $\boldsymbol{k}=|\boldsymbol{n}| \hat{\boldsymbol{k}}=|\boldsymbol{n}|\left(-\hat{n}_{2}, \hat{n}_{1}\right)$.

The operator $\mathcal{S}^{\mathbf{n}}$ becomes

$$
\mathcal{S}^{\mathbf{n}}=\left[\begin{array}{cccc}
\alpha & 1 & 0 & \alpha \\
\alpha\left(u+c \hat{n}_{1}\right) & u & -\hat{n}_{2} & \alpha\left(u-c \hat{n}_{1}\right) \\
\alpha\left(v+c \hat{n}_{2}\right) & v & \hat{n}_{1} & \alpha\left(v-c \hat{n}_{2}\right) \\
\alpha(H+c \boldsymbol{u} \cdot \hat{\boldsymbol{n}}) & \frac{1}{2} q^{2} & \boldsymbol{u} \cdot \hat{\boldsymbol{k}} & \alpha(H-c \boldsymbol{u} \cdot \hat{\boldsymbol{n}})
\end{array}\right] .
$$

Here $\boldsymbol{u}=(u, v)$ is the convective velocity vector and $q^{2}=\boldsymbol{u} \cdot \boldsymbol{u}$, and we have introduced the constant $\alpha=1 /(2 c)$ and the specific stagnation enthalpy $H=(E+p) / \rho$. In $\mathcal{S}^{\mathbf{n}}$ all physical variables refer to the state $\boldsymbol{q}_{0}$ around which we have linearized. The characteristic variables $\boldsymbol{R}^{\mathbf{n}}=\left(\mathcal{S}^{\mathbf{n}}\right)^{-1} \boldsymbol{q}$ are

$$
\boldsymbol{R}^{\mathbf{n}}=\left[\begin{array}{c}
\boldsymbol{m} \cdot \hat{\boldsymbol{n}}+\frac{\gamma-1}{c_{0}}\left(E+\frac{1}{2} \rho q_{0}^{2}-\rho \boldsymbol{u}_{0} \cdot \boldsymbol{u}\right) \\
\rho-\frac{\gamma-1}{c_{0}^{2}}\left(E+\frac{1}{2} \rho q_{0}^{2}-\rho \boldsymbol{u}_{0} \cdot \boldsymbol{u}\right) \\
\boldsymbol{m} \cdot \hat{\boldsymbol{k}} \\
-\boldsymbol{m} \cdot \hat{\boldsymbol{n}}+\frac{\gamma-1}{c_{0}}\left(E+\frac{1}{2} \rho q_{0}^{2}-\rho \boldsymbol{u}_{0} \cdot \boldsymbol{u}\right)
\end{array}\right]
$$

where we have the linearized momentum $\boldsymbol{m}=\rho\left(\boldsymbol{u}-\boldsymbol{u}_{0}\right)$. The corresponding eigenvalues, i.e., the advective velocity of the characteristic waves, become

$$
\lambda_{1}^{\mathbf{n}}=\boldsymbol{u}_{0} \cdot \boldsymbol{n}+c_{0}|\boldsymbol{n}|, \quad \lambda_{2}^{\mathbf{n}}=\lambda_{3}^{\mathbf{n}}=\boldsymbol{u}_{0} \cdot \boldsymbol{n}, \lambda_{4}^{\mathbf{n}}=\boldsymbol{u}_{0} \cdot \boldsymbol{n}-c_{0}|\boldsymbol{n}| .
$$

In deriving the proper well-posed boundary operator, we introduced the viscous correction vector having the explicit entries

$$
\boldsymbol{G}^{\mathbf{n}}=|\boldsymbol{n}|\left[\begin{array}{c}
\frac{k_{0}(\gamma-1)}{2 \operatorname{Pr} \rho_{0}} \boldsymbol{V}_{1} \cdot \hat{\boldsymbol{n}}+\frac{\lambda_{0}+2 \mu_{0}}{2 \mu_{0}} \boldsymbol{V}_{2} \cdot \hat{\boldsymbol{n}}-\frac{\lambda_{0}+\mu_{0}}{2 \rho_{0}} \boldsymbol{V}_{3} \cdot \hat{\boldsymbol{k}} \\
-\frac{\kappa_{0}(\gamma-1)}{2 \operatorname{Prc} c_{0}} \boldsymbol{V}_{1} \cdot \hat{\boldsymbol{n}} \\
-\frac{\mu_{0}}{\rho_{0}} \boldsymbol{V}_{3} \cdot \hat{\boldsymbol{n}}+\frac{\lambda_{0}+\mu_{0}}{4 \rho_{0}} \boldsymbol{V}_{2} \cdot \hat{\boldsymbol{k}} \\
\frac{k_{0}(\gamma-1)}{2 \operatorname{Pr} \rho_{0}} \boldsymbol{V}_{1} \cdot \hat{\boldsymbol{n}}-\frac{\lambda_{0}+2 \mu_{0}}{2 \rho_{0}} \boldsymbol{V}_{2} \cdot \hat{\boldsymbol{n}}+\frac{\lambda_{0}+\mu_{0}}{2 \rho_{0}} \boldsymbol{V}_{3} \cdot \hat{\boldsymbol{k}}
\end{array}\right],
$$

where we have the vectors

$$
\boldsymbol{V}_{1}=\nabla R_{1}^{\mathbf{n}}+\nabla R_{4}^{\mathbf{n}}-\frac{2 c_{0}}{(\gamma-1)} \nabla R_{2}^{\mathbf{n}}, \quad \boldsymbol{V}_{2}=\nabla R_{1}^{\mathbf{n}}-\nabla R_{4}^{\mathbf{n}}, \quad \boldsymbol{V}_{3}=\nabla R_{3}^{\mathbf{n}} .
$$

Acknowledgments. The author appreciates many enlightening discussions with Prof. D. Gottlieb and Dr. C. Quillen, Brown University. Gratitude is also extended toward Prof. C. D. Pruett and Dr. C. L. Streett for permitting access to and use of their spectral boundary layer code and to Prof. D. A. Kopriva, Florida State University, for establishing the contact.

\section{REFERENCES}

[1] J. S. Hesthaven and D. Gottlieb, A stable penalty method for the compressible Navier-Stokes equations. I. Open boundary conditions, SIAM J. Sci. Comput., 17 (1996), pp. 579-612. 
[2] J. S. Hesthaven, A stable penalty method for the compressible Navier-Stokes equations. II. One dimensional domain decomposition schemes, SIAM J. Sci. Comput., 18 (1997), pp. 658685 .

[3] D. Kopriva, Multidomain spectral solution of the Euler gas-dynamics equations, J. Comput. Phys., 96 (1991), pp. 428-450.

[4] D. Kopriva AND J. H. Kolias, A conservative staggered-grid Chebyshev multidomain method for compressible flows. J. Comput. Phys., 125 (1996), pp. 244-261.

[5] A. Quarteroni, Domain decomposition methods for systems of conservation laws, SIAM J. Sci. Statist. Comput., 11 (1990), pp. 1029-1052.

[6] J. G. Giannakouros, D. Sidilkover and G. E. Karniadakis, Spectral element-FCT method for the one- and two-dimensional compressible Euler equations, Comput. Methods Appl. Mech. Engrg., 116 (1994), pp. 113-121.

[7] D. Kopriva, A multi-domain spectral method for viscous compressible flows, AIAA J., 31 (1993), pp. 3376-3384.

[8] P. Hanley, A strategy for the efficient simulation of viscous compressible flows using a multidomain pseudospectral method, J. Comput. Phys., 108 (1993), pp. 153-158.

[9] D. Kopriva, Multidomain spectral solution of compressible viscous flows, J. Comput. Phys., 115 (1994), pp. 184-199.

[10] I. Lomtev, C. B. Quillen, And G. Karniadakis, Spectral/hp Methods for Viscous Compressible Flows on Unstructured 2D Meshes, Center for Fluid Mechanics Report \# 96-12, Brown University, Providence, RI, 1996.

[11] D. Funaro And D. Gottlieb, A new method of imposing boundary conditions in pseudospectral approximations of hyperbolic equations, Math. Comp., 51 (1988), pp. 599-613.

[12] D. Funaro and D. GotTlieb, Convergence results for pseudospectral approximations of hyperbolic systems by a penalty-type boundary treatment, Math. Comp., 57 (1991), pp. 585-596.

[13] L. H. Loomis and S. Sternberg, Advanced Calculus, Addison-Wesley, Reading, MA, 1968.

[14] W. J. Gordon And C. A. Hall, Transfinite element methods: Blending-function interpolation over arbitrary curved element domains, Numer. Math., 21 (1973), pp. 109-129.

[15] W. J. Gordon and C. A. Hall, Construction of curvilinear co-ordinate systems and applications to mesh generation, Internat. J. Numer. Meth. Engrg., 7 (1973), pp. 461-477.

[16] D. Funaro, Polynomial Approximation of Differential Equations, Springer-Verlag, New York, 1992.

[17] C. Canuto, M. Y. Hussaini, A. Quarteroni, and T. A. Zang, Spectral Methods in Fluid Dynamics, Springer Series in Computational Physics, Springer-Verlag, New York, 1988.

[18] L. Dettori And B. Yang, On the Chebyshev penalty method for parabolic and hyperbolic equations, Math. Anal. Numer. Anal. (M²AN), 30 (1996), pp. 907-920.

[19] W. S. Don And D. GotTlieb, The Chebyshev-Legendre Method: Implementing Legendre Methods on Chebyshev Grids, SIAM J. Numer. Anal., 31 (1994), pp. 1519-1534.

[20] H. Schlichting, Boundary-Layer Theory, 7th ed., McGraw-Hill, New York, 1979.

[21] H. O. Kreiss and J. Lorenz, Initial-Boundary Value Problems and the Navier-Stokes Equations, Series in Pure and Applied Mathematics, Academic Press, San Diego, CA, 1989.

[22] R. F. Warming, R. M. Beam, and B. J. Hyett, Diagonalization and simultaneous symmetrization of the gas-dynamics matrices, Math. Comp., 29 (1975), pp. 1037-1045.

[23] R. A. Horn and C. A. Johnson, Matrix Analysis, Cambridge University Press, Cambridge, 1985.

[24] J. C. Strikwerda, Initial boundary value problems for incompletely parabolic systems, Comm. Pure Appl. Math., 30 (1977), pp. 797-822.

[25] S. Abarbanel and D. GotTlieb, Optimal time splitting for two- and three-dimensional Navier-Stokes equations with mixed derivatives, J. Comput. Phys., 41 (1981), pp. 1-33.

[26] C. D. Pruett and C. L. Streett, A spectral collocation for compressible, non-similar boundary layers, Internat. J. Numer. Methods Fluids, 13 (1991), pp. 713-737.

[27] P. Fischer And D. Gottlieb, On the optimal number of subdomains for hyperbolic problems on parallel computers, J. Supercomput. Appl., 11 (1997), pp. 65-76.

[28] A. Harten, On the symmetric form of systems of conservation laws with entropy, J. Comput. Phys., 49 (1983), pp. 151-164. 